\title{
Local Entropy Generation Analysis
}

M. K. Drost

M. D. White

February 1991

Prepared for the U.S. Department of Energy

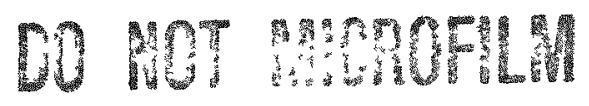

COVER under Contract DE-AC06-76RLO 1830

Pacific Northwest Laboratory

Operated for the U.S. Department of Energy

by Battelle Memorial Institute 


\title{
DISCLAIMER
}

This report was prepared as an account of work sponsored by an agency of the United States Government. Neither the United States Government nor any agency thereof, nor Battelle Memorial Institute, nor any of their employees, makes any warranty, expressed or implied, or assumes any legal liability or responsibility for the accuracy, completeness, or usefulness of any information, apparatus, product, or process disclosed, or represents that its use would not infringe privately owned rights. Reference herein to any specific commercial product, process, or service by trade name, trademark, manufacturer, or otherwise does not necessarily constitute or imply its endorsement, recommendation, or favoring by the United States Government or any agency thereof, or Battelle Memorial Institute. The views and opinions of authors expressed herein do not necessarily state or reflect those of the United States Government or any agency thereof.

\author{
PACIFIC NORTHWEST LABORATORY \\ operated by \\ BATTELLE MEMORIAL INSTITUTE \\ for the \\ UNITED STATES DEPARTMENT OF ENERGY \\ under Contract DE-AC06-76RLO 1830
}

Printed in the United States of America

Available to DOE and DOE contractors from the

Office of Scientific and Technical Information, P.O. Box 62, Oak Ridge, TN 37831;

prices available from (615) 576-8401. FTS 626-8401.

Available to the public from the National Technical Information Service, U.S. Department of Commerce, 5285 Port Royal Rd., Springfield, VA 22161. 


\section{DISCLAIMER}

This report was prepared as an account of work sponsored by an agency of the United States Government. Neither the United States Government nor any agency Thereof, nor any of their employees, makes any warranty, express or implied, or assumes any legal liability or responsibility for the accuracy, completeness, or usefulness of any information, apparatus, product, or process disclosed, or represents that its use would not infringe privately owned rights. Reference herein to any specific commercial product, process, or service by trade name, trademark, manufacturer, or otherwise does not necessarily constitute or imply its endorsement, recommendation, or favoring by the United States Government or any agency thereof. The views and opinions of authors expressed herein do not necessarily state or reflect those of the United States Government or any agency thereof. 


\section{DISCLAIMER}

Portions of this document may be illegible in electronic image products. Images are produced from the best available original document. 
M. K. Drost

M. D. White

February 1991

Prepared for the U.S. Department of Energy under Contract DE-AC06-76RLO 1830

Pacific Northwest Laboratory Richland, Washington 99352 



\section{SUMMARY}

Second law analysis techniques have been widely used to evaluate the sources of irreversibility in components and systems of components but the evaluation of local sources of irreversibility in thermal processes has received little attention. While analytical procedures for evaluating local entropy generation have been developed, applications have been limited to fluid flows with analytical solutions for the velocity and temperature fields. The analysis of local entropy generation can be used to evaluate more complicated flows by including entropy generation calculations in a computational fluid dynamics (CFD) code. The research documented in this report consists of incorporating local entropy generation calculations in an existing CFD code and then using the code to evaluate the distribution of thermodynamic losses in two applications: an impinging jet and a magnetic heat pump.

The results of the study demonstrate three general conclusions concerning numerical analysis of local entropy generation:

- Local entropy generation analysis proved to be a powerful tool for improving the design of heat transfer devices. Local entropy generation analys is provided the means for optimizing the design of both an impinging jet heat exchanger and the regenerator for a magnetic heat pump. In the case of the regenerator evaluation, local entropy analysis suggested the use of flow disrupters for enhancing heat transfer by clearly identifying the real sources of thermodynamic performance losses.

- The integration of local entropy generation calculations into an existing CFD code proved to be straightforward and did not significantly increase the demand on computational resources. The comparison of numerical and analytical results for a simple benchmarking problem was successful.

- When evaluating heat transfer enhancements, a second law figure-of-merit must be used because traditional figures-of-merit do not account for the difference in the relative importance between the heat transfer losses and the pressure drop losses. This resulted in the traditional figure-ofmerit indicating that the use for flow disrupters in the regenerator of a magnetic heat pump was unattractive when local entropy generation analys is clearly indicated that the use of flow disrupters was an extremely attractive method of improving performance. 
The first application of local entropy generation analysis consisted of evaluating local entropy generation in a fluid jet impinging on a heated surface. Impingement heat transfer has important industrial applications and improving the process by thermodynamic analysis should be of interest. The results of the analysis showed:

- Numerical calculations allow the investigation of the structure of local entropy generation in thermal processes. The impinging jet problem is an example. The results show that for the sample problem, entropy generation is concentrated in the boundary layer. To a great extent, understanding the boundary layer will allow an understanding of local entropy generation. This will not necessarily be true for different geometries and more complicated processes.

- The results of the impinging jet problem identified a jet Reynolds number that minimizes entropy generation. This information would be useful to a designer trying to minimize the thermodynamic performance losses in a jet impingement heat exchanger.

The second application involved an analysis of local entropy generation occurring in the regenerator of a magnetic heat pump. The magnetic heat pump is being considered by the U.S. Department of Energy for applications in the cryogenic industry. The attractiveness of the magnetic heat pump is, to a great extent, determined by the effectiveness of its regenerator. While lumped parameter second law analysis has been used to evaluate the magnetic heat pump regenerator, local entropy generation analys is has not been applied to this application. The results of the local entropy generation analys is of the magnetic heat pump regenerator include:

- In the base case regenerator design, heat transfer related entropy generation dominates viscous dissipation losses. This suggests that the design should be altered to improve heat transfer, even if the alterations result in a substantial increase in viscous dissipation losses.

- It is important to maintain balanced flow in the regenerator (where the thermal capacity of the magnetic material flow approximately equals the thermal capacity of the regenerator fluid flow). Substantial deviation from balanced flow conditions can result in a significant increase in thermodynamic losses.

- Entropy generation caused by conduction in the magnetic material is a significant loss mechanism and must be included explicitly in any performance evaluation. 
- The addition of a flow disrupter can significantly improve the performance of the regenerator. The optimum disrupter design will block more than $75 \%$ of the flow area.

The evaluation of the flow disrupter concept illustrates the importance of using second law analysis for evaluating heat transfer enhancements. Conventional figures-of-merit, such as the efficiency index, suggest that the flow disrupter is not an attractive enhancement technique, while local entropy generator analysis shows that the use of flow disrupters is very attractive. The reason for the different conclusions is related to the magnitudes of the different loss mechanisms. In the base case magnetic heat pump regenerator design, thermal losses are two orders of magnitude larger than the pressure drop 1osses. Consequently, an enhancement technique that reduces heat transfer losses is attractive, even if it results in a substantial increase in pressure drop losses. The efficiency index is a first law figure-of-merit and does not account for the difference in relative importance between the heat transfer losses and the pressure drop losses.

In the course of the study it was discovered that local entropy generation analysis could be a sensitive indicator of numerical entropy generation. The results of numerical experimentation suggest that the numerical calculation of local entropy generation and its comparison with a surface integration of entropy flows provide a measure of numerical entropy generation that can be compared to natural entropy generation to judge the validity of the numerical calculation. Further investigation of the use of local entropy generation analysis for evaluating numerical entropy generation will be required to confirm this finding. 



\section{NOMENCLATURE}

\begin{tabular}{|c|c|}
\hline A & flow area, $\mathrm{m}^{2}$ \\
\hline C & ratio of thermal capacities, $\mathrm{H}_{\mathrm{mm}} \mathrm{CPmm} /\left(\mathrm{m} r \mathrm{C} \mathrm{Pp}_{\mathrm{f}}\right)$ \\
\hline$C_{p}$ & specific heat, $\mathrm{kJ} / \mathrm{kg} \mathrm{K}$ \\
\hline $\mathrm{d}$ & flow path width, m \\
\hline D & hydraulic diameter, $m$ \\
\hline f & friction factor \\
\hline$h$ & specific enthalpy, $\mathrm{kJ} / \mathrm{kg}$ \\
\hline$H$ & disrupter height, m \\
\hline k & thermal conductivity, $w / m k$ \\
\hline $\mathrm{L}$ & regenerator length, m \\
\hline$\dot{\mathrm{m}}$ & mass flow rate, $\mathrm{kg} / \mathrm{s}$ \\
\hline N & augmentation entropy generation number \\
\hline P & pressure, $\mathrm{N} / \mathrm{m}^{2}$ \\
\hline $\mathrm{Pe}$ & Peclet number \\
\hline$\dot{q}_{0}^{\prime}$ & surface heat flux per unit length, $\mathrm{w} / \mathrm{m}$ \\
\hline$\dot{q} \ddot{0}$ & surface heat flux per unit area, $\mathrm{W} / \mathrm{m}^{2}$ \\
\hline$r$ & radius, $m$ \\
\hline R & disrupter spacing, m \\
\hline $\operatorname{Re}$ & Reynolds number based on flow path width \\
\hline$r_{0}$ & conduit radius, $\mathrm{m}$ \\
\hline$s$ & specific entropy, $\mathrm{kJ} / \mathrm{kg} \mathrm{k}$ \\
\hline gen & local entropy generation rate, $\mathrm{W} / \mathrm{m}^{3} \mathrm{~K}$ \\
\hline & $\begin{array}{l}\text { local entropy generation rate caused by thermal conduction } \\
W / \mathrm{m}^{3} \mathrm{~K}\end{array}$ \\
\hline & total local entropy generation rate, $W / \mathrm{m}^{3} \mathrm{~K}$ \\
\hline & $\begin{array}{l}\text { local entropy generation rate caused by viscous dissipation, } \\
W / \mathrm{m}^{3} \mathrm{~K}\end{array}$ \\
\hline & integrated entropy generation rate, $W / K$ \\
\hline
\end{tabular}




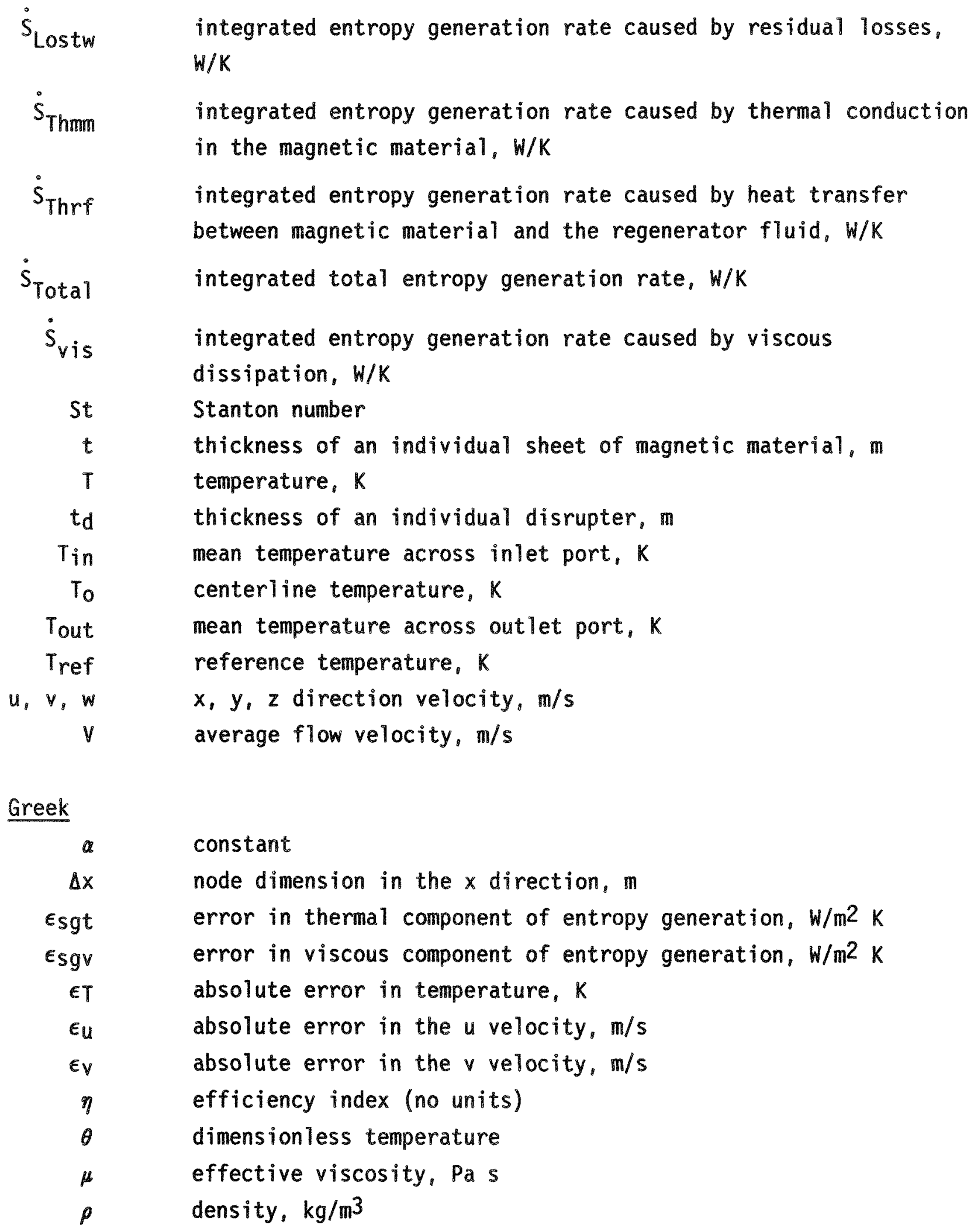

integrated entropy generation rate caused by thermal conduction in the magnetic material, $W / K$

integrated entropy generation rate caused by heat transfer between magnetic material and the regenerator fluid, $W / K$ integrated total entropy generation rate, $\mathrm{W} / \mathrm{K}$

integrated entropy generation rate caused by viscous dissipation, $W / K$

\section{Stanton number}

thickness of an individual sheet of magnetic material, m temperature, $k$ thickness of an individual disrupter, $m$ mean temperature across inlet port, $K$ centerline temperature, $k$ mean temperature across outlet port, $k$ reference temperature, $k$ $x, y, z$ direction velocity, $\mathrm{m} / \mathrm{s}$ average flow velocity, $\mathrm{m} / \mathrm{s}$

Greek

$a$

$\Delta x$

$\epsilon_{\text {sgt }}$

$\epsilon_{\text {sgv }}$

$\epsilon \mathrm{T}$

$\epsilon_{u}$

$\varepsilon_{y}$

$\eta$

$\theta$

$\mu$

\section{constant}

node dimension in the $x$ direction, $m$

error in thermal component of entropy generation, $w / \mathrm{m}^{2} \mathrm{~K}$

error in viscous component of entropy generation, $W / m^{2} K$

absolute error in temperature, $k$

absolute error in the $u$ velocity, $\mathrm{m} / \mathrm{s}$

absolute error in the $v$ velocity, $\mathrm{m} / \mathrm{s}$

efficiency index (no units)

dimensionless temperature

effective viscosity, $\mathrm{Pa} s$

$\rho$

density, $\mathrm{kg} / \mathrm{m}^{3}$ 
$\begin{array}{ll}v & \text { specific volume, } \mathrm{m}^{3} / \mathrm{kg} \\ \phi & \text { irreversibility distribution ratio }\end{array}$

Subscripts

e

i

j

mm

$p$

rf

related to performance with enhancement

$x$ direction node index

$y$ direction node index

related to magnetic materials

related to performance without enhancement

related to regenerator fluid 



\section{CONTENTS}

SUMMARY

NOMENCLATURE ........................... v vii

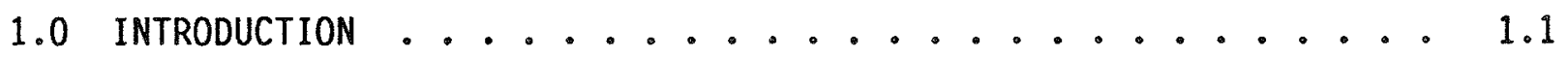

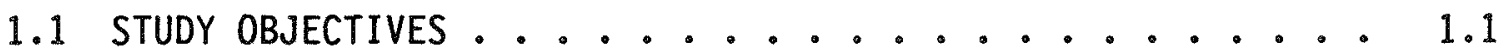

1.2 PREVIOUS RESEARCH . . . . . . . . . . . 1.2

2.0 NUMERICAL CALCULATION OF LOCAL ENTROPY GENERATION . . . . . . 2.1

2.1 LOCAL ENTROPY GENERATION . . . . . . . . . . 2.1

2.2 NUMERICAL CALCULATION OF LOCAL ENTROPY GENERATION . . . 2.2

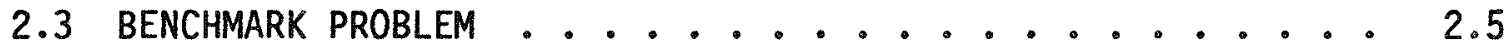

3.0 LOCAL ENTROPY GENERATION IN AN IMPINGING JET $\ldots . \cdots$

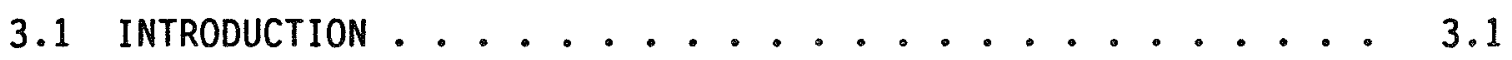

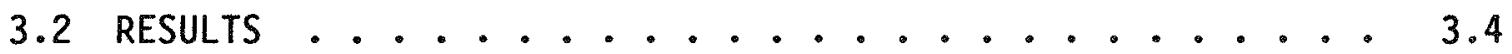

3.2.1 Integrated Entropy Generation . . . . . . . 3.5

3.2.2 Local Entropy Generation . . . . . . . . . 3.7

3.2.3 Error Analysis ............... 3.11

4.0 LOCAL ENTROPY GENERATION IN A MAGNETIC HEAT PUMP REGENERATOR • • 4.1

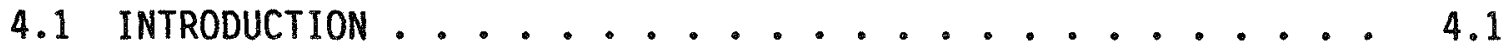

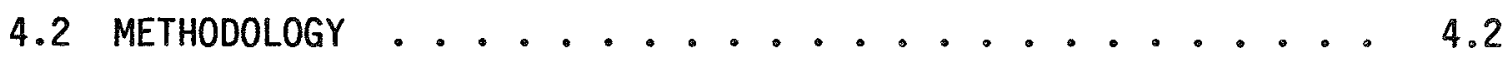

4.2.1 Parametric Analysis ............ 4.4

4.2.2 Evaluation of the Flow Disrupter Concept . . . . 4.7

4.2.3 Evaluation of Heat Exchanger Thermodynamic

Figures-of-Merit ........... 4.8

4.3 RESULTS ....................... 4.8

4.3.1 Parametric Analys is of Base Case Design . . . . . 4.9 
4.3.2 Analysis of Flow Disrupter Concept .......4 4.11

4.3.3 Figures-of-Merit for Heat Exchanger Analysis ... 4.13

5.0 EVALUATION OF NUMERICAL ERRORS USING LOCAL ENTROPY GENERATION

ANALYSIS ......................... 5.1

5.1 THE ENTROPY BALANCE .................... 5.1

5.2 RESULTS OF SAMPLE SIMULATIONS ........... 5.3

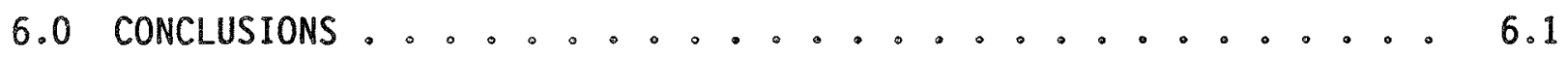

7.0 REFERENCES ........................ 7.1 


\section{FIGURES}

1 Entropy Generation for Laminar Flow with Constant Heat Flux . . . . 2.6

2 Impinging Jet . . . . . . . . . . . . . . . 3.1

3 Computational Grid for the Impinging Jet Problem . . . . . . 3.3

4 Viscous Component of Local Entropy Generation W/m $\mathrm{K}$. . . . . 3.8

5 Thermal Component of Local Entropy Generation W/m K . . . . . 3.9

6 Total Local Entropy Generation W/m $\mathrm{m}^{3}$............ 3.10

7 Viscous Component of Local Entropy Generation W/m $\mathrm{K}$. . . . . 3.12

8 Thermal Component of Local Entropy Generation W/m $\mathrm{m}^{3}$. . . . . 3.13

9 Rotary Magnetic Heat Pump . . . . . . . . . . . . . . 4.3

10 Regenerator Flow Path Model . . . . . . . . . . . . 4.4

11 Flow Disrupter . . . . . . . . . . . . . . . 4.7

12 Computational Grid for IAHR Test Problem . . . . . . . . . 5.6

13 Outlet Profile for Advecting Variable . . . . . . . . . . 5.7 


\section{TABLES}

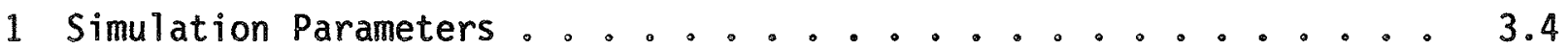

2 Integrated Entropy Generation Rates ............ 3.6

3 Relation Between Primitive Errors and Entropy Errors ..... 3.14

4 Integrated Entropy Generation as a Function of Regenerator Length ....................... 4.9

5 Integrated Entropy Generation as a Function of the Ratio of micp . . 4.10

6 Integrated Energy Generation as a Function of Regenerator

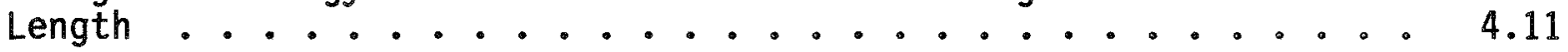

7 Integrated Local Entropy Generation for a Simple Flow Disrupter Design as a Function of Blocked Flow Area . . . . . 4.12

8 Comparison of Integrated Local Entropy Generation with the Results of the Entropy Balance Equation for Poiseuille and Entrance Flow .................... 5.4

9 Comparison of Integrated Local Entropy Generation with the Results of the Entropy Balance Equation for IAHR Test Problem . . 5.8 


\section{LOCAL ENTROPY GENERATION ANALYSIS}

\subsection{INTRODUCTION}

A large number of studies have shown that the valid measure of thermodynamic performance of a device or process is its irreversibility (Van Wylen and Sonntag 1971, Bejan 1982, Gaggioli 1983). Second law analys is techniques have been widely used to evaluate the sources of irreversibility in components and systems of components but the evaluation of local sources of irreversibility in thermal processes has received limited attention. While analytical procedures for evaluating local entropy generation were published in 1979 (Bejan 1979a), applications have been limited to fluid flows with analytical solutions for the velocity and temperature fields. A recent review of second law analysis techniques applicable to basic research in the thermal sciences recommended that entropy generation calculations be included in computational fluid dynamics (CFD) codes to allow the evaluation of local entropy generation in more complicated thermal phenomena (Drost and Zaworski 1988). The research documented here is in response to that recommendation and consists of incorporating local entropy generation calculations in an existing CFD code and then using the code to evaluate local entropy generation in two applications: an impinging jet heat exchanger and a regenerator for a magnetic heat pump.

\subsection{STUDY OBJECTIVES}

The research documented in this report focused on developing and testing numerical procedures for determining local entropy generation. Specific objectives included:

- Incorporate calculation of local entropy generation in an existing CFD code.

- Benchmark the entropy generation calculations by comparing numerical results with analytical calculations for laminar pipe flow.

- Use the modified CFD code to investigate the sources, magnitudes, and distribution of local entropy generation in two applications: an impinging heat exchanger and a regenerator for a magnetic heat pump. 
- Evaluate the usefulness of local entropy generation calculations in the design of thermal system components.

\subsection{PREVIOUS RESEARCH}

The application of partial differential equations for local entropy generation in a convecting fluid was proposed by Bejan (1979a), and he has applied this procedure to the evaluation of local entropy generation in several simple flow situations where analytical solutions were available for the velocity and temperature fields (Bejan 1979a, 1982). Bejan's work is the only example of local entropy generation calculations identified in the literature.

While the calculation of local entropy generation from the velocity and temperature fields has been limited, a number of investigators have used a lumped parameter approach to calculate entropy generation. Bejan has evaluated entropy generation in internal conduit flow, external flow, and in insulation systems using lumped parameters (Bejan 1979a, 1979b, 1982). Dunbar (1982) has applied a one-dimensional lumped parameter analysis to evaluation of a fuel cell, while Hutchinson and Lyke (1987) have used a similar approach to investigate irreversibilities in a Stirling engine regenerator. Two recent papers have presented one-dimensional lumped parameter evaluations of local entropy generation in heat exchangers (Liang and Kuehn 1988, El-Sayed 1988).

While one researcher has suggested that calculation of local entropy generation can be used to evaluate the importance of numerical entropy generation (Argrow, Emanual, and Rasmussen 1987), an extensive literature review did not identify any previous studies that actually incorporated entropy generation relationships into a CFD code and then used the code to evaluate an important thermodynamic process. 


\subsection{NUMERICAL CALCULATION OF LOCAL ENTROPY GENERATION}

One goal of thermal design should be to identify and minimize the thermodynamic irreversibilities. It can be shown that the minimization of entropy generation also results in the maximum reduction in irreversibility (Bejan 1982). The development of improved thermal designs will be enhanced by the ability to clearly identify the source and location of entropy generation. Systems of individual components can be optimized from estimates of net entropy generation for complete components but the development of novel components and processes should benefit from knowing the distribution and sources of entropy generation on a local level.

\subsection{LOCAL ENTROPY GENERATION}

For a convecting, isotropic, and Newtonian fluid, entropy generation rate per unit volume is given by Equation (1) for three-dimensional rectangular coordinates (Bejan 1982).

$$
\dot{S}_{\text {gen }}^{\prime \prime \prime}=\dot{S}_{\text {Th }}^{\prime \prime \prime}+\dot{S}_{\text {vis }}^{\prime \prime \prime}
$$

where

$$
\begin{aligned}
& \dot{S}_{T h}^{\prime \prime \prime}=\frac{k}{T^{2}}\left[\left(\frac{\partial T}{\partial x}\right)^{2}+\left(\frac{\partial T}{\partial y}\right)^{2}+\left(\frac{\partial T}{\partial z}\right)^{2}\right] \\
& \dot{S}_{v i s}^{\prime \prime \prime}=\frac{\mu}{T}\left\{2\left[\left(\frac{\partial u}{\partial x}\right)^{2}+\left(\frac{\partial v}{\partial y}\right)^{2}+\left(\frac{\partial w}{\partial z}\right)^{2}\right]+\left(\frac{\partial u}{\partial y}+\frac{\partial v}{\partial x}\right)^{2}+\left(\frac{\partial u}{\partial z}+\frac{\partial w}{\partial x}\right)^{2}+\left(\frac{\partial v}{\partial z}+\frac{\partial w}{\partial y}\right)^{2}\right\}
\end{aligned}
$$

Equation (2) is entropy generation caused by heat transfer across a finite temperature difference, whereas Equation (3) is the local entropy generation caused by viscous dissipation. When analytical expressions are available for the temperature and velocity fields, Equation (1) can be evaluated to yield the 
local entropy generation. Total entropy generation can be determined by integrating Equation (1) over the region (volume) of interest. Most practical problems are sufficiently complex that analytical solutions do not exist. Alternatively, a CFD computer code can be used to numerically predict the velocity and temperature fields. This information can then be used to numerically calculate entropy generation.

Equation (1) is valid for both laminar and turbulent flows: and the effective thermal conductivity is the sum of the molecular thermal conductivity and the eddy thermal conductivity, and the effective viscosity is the sum of the molecular viscosity and the eddy diffusivity. Local entropy generation rates are, therefore, dependent on the local spatial gradients of temperature and velocity and on the absolute local values of temperature, effective thermal conductivity, and effective viscosity. These quantities were estimated locally for the fluid flow and convective heat transfer problems considered in this study, with the finite-difference based TEMPEST computer code(a) (Trent, Eyler and Budden 1989a, b).

\subsection{NUMERICAL CALCULATION OF LOCAL ENTROPY GENERATION}

The method for computing local entropy generation consisted of substituting the finite difference formulations for the partial derivative terms of Equation (1) into TEMPEST and evaluating the resulting expression after each simulation time step. TEMPEST is a three-dimensional, timedependent computer program for hydrothermal analysis and it generates the local velocity and temperature gradients required to compute the local entropy generation rate.

The evaluation of local entropy generation throughout the computational domain is decoupled from the computation of the temperature, pressure, velocity, turbulent kinetic energy, and the dissipation of turbulent kinetic energy. Conserving the first-order accuracy of the finite differencing scheme used in the TEMPEST code, the entropy generation equation was also differenced

(a) Computer code developed and marketed by Pacific Northwest Laboratory, Richland, Washington. 
to first-order accuracy. The difference expressions for 3 of the 12 partial differential terms in Equation (1) are presented below:

$$
\begin{aligned}
\left(\frac{\partial T}{\partial x}\right)^{2} & =\left\{\frac{\left(\Delta x_{i-1}+\Delta x_{i}\right) T_{i+1}-\left(\Delta x_{i+1}+\Delta x_{i}\right) T_{i-1}+\left(\Delta x_{i+1}-\Delta x_{i-1}\right) T_{i}}{2\left(\Delta x_{i+1}+\Delta x_{i}\right)\left(\Delta x_{i-1}+\Delta x_{i}\right)}\right\}^{2} \\
\left(\frac{\partial u}{\partial x}\right)^{2} & =\left(\frac{u_{i}-u_{i-1}}{\Delta x_{i}}\right)^{2} \\
\left(\frac{\partial v}{\partial x}\right)^{2} & =\left\{\left[\frac{\left(\Delta x_{i-1}+\Delta x_{i}\right)\left(v_{i+1, j}+v_{i+1, j-1}\right)}{2\left(\Delta x_{i+1}+\Delta x_{j}\right)\left(\Delta x_{i-1}+\Delta x_{j}\right)}\right]-\left[\frac{\left(\Delta x_{i+1}+\Delta x_{i}\right)\left(v_{i-1, j}+v_{i-1, j-1}\right)}{2\left(\Delta x_{i+1}+\Delta x_{i}\right)\left(\Delta x_{i-1}+\Delta x_{j}\right)}\right]\right. \\
& \left.+\left[\frac{\left(\Delta x_{i+1}-\Delta x_{i-1}\right)\left(v_{j}+v_{j-1}\right)}{2\left(\Delta x_{i+1}+\Delta x_{i}\right)\left(\Delta x_{i-1}+\Delta x_{j}\right)}\right]\right\}^{2}
\end{aligned}
$$

The subscripts $i$ and $j$ are indices for the positive $x$ and $y$ Cartesian coordinate directions, respectively. The subscripting convention is to suppress all indices except for those indices that differ from the local cell $(i, j, k)$. The finite difference expression for entropy generation reflects the staggered grid arrangement used in TEMPEST where the scalar variables are cell centered, and the velocities are positioned on the cell faces. The $u$ velocity associated with the $i, j, k$ cell is, for example, positioned on the face between the $i, j, k$ and $i+1, j, k$ cells. The same indexing logic holds for the $y$ and $z$ Cartesian directions.

TEMPEST solves the three conservation equations governing mass, momentum, and energy, subject to several important assumptions. The fluid is modeled as single-phase, incompressible, and Newtonian. The Boussinesq approximation is assumed and the heating contribution caused by viscous dissipation is eliminated from the energy equation. These assumptions combine to yield a loosely-coupled momentum and energy equations where the coupling is through the thermodynamic state relationship. The coupling is important for simulations where natural convection is significant. 
The turbulent flow conservation equations are time-averaged and Reynolds stresses are incorporated through appropriate eddy viscosity models. In the current version of TEMPEST, turbulent flow Reynolds stresses are modeled through an effective viscosity. The Prandtl-Kolmogorov hypothes is is used to relate the effective viscosity to a velocity and a length scale. In this approach, transport equations for turbulent kinetic energy and dissipation of turbulent kinetic energy are solved to determine the effective turbulent viscosity. The turbulent viscosity is then employed to compute the turbulent (eddy) thermal conductivity.

The TEMPEST solution procedure is a semi-implicit time marching finite-difference approach with all governing equations solved sequentially. For each time step, the momentum equations are solved explicitly while the solution of the pressure equations uses an implicit formulation. Temperature, turbulent kinetic energy, dissipation of turbulent kinetic energy, and other scalar transport equations are solved using an implicit continuation procedure. The solution includes three phases. During the first phase, the three momentum equations are advanced in time to obtain approximations to the velocity field based on previous time step values of the pressure and density fields. The initial velocity fields satisfy the momentum equation, but not necessarily the continuity equation. The implicit phase computes corrections to the velocity and pressure fields such that the adjusted quantities satisfy the continuity equation. With the implicit phase velocities computed, the scalar phase updates (time advances) the values of temperature and other scalar quantities. The solution is advanced in time by repeated application of the three solution phases described above.

It might appear that calculation of entropy generation could be included in the scalar phase; however, unlike the other scalar quantities, the entropy generation does not influence the solution of the momentum equation. The entropy generation quantities should, therefore, be considered as a post scalar phase.

For the steady-state solutions presented in this study, the concept of time stepping is somewhat artificial because the solution is brought to steady-state from some arbitrary initial guess through a series of artificial transients. 


\subsection{BENCHMARK PROBLEM}

The procedure for calculating local entropy generation was benchmarked by comparing the numerical results with an analytical solution for laminar flow in a circular duct with a constant wall heat flux. The problem was specifically structured so that the entropy generation caused by viscous dissipation and thermal gradients were of the same order. The benchmark problem involved the flow of $150^{\circ} \mathrm{C}$ helium at an average velocity of $8.75 \mathrm{~m} / \mathrm{s}$, through a circular duct with a diameter of $0.05 \mathrm{~m}$. The duct was assumed to have a constant wall heat flux of $45 \mathrm{~W} / \mathrm{m}^{2}$. The properties of helium were held constant for both the analytical and numerical evaluations. The analytical solution for the velocity profile and temperature profile are presented in Equations (7) and (8) (Kays and Crawford 1980).

$$
\begin{aligned}
& u=2 v\left(1-\frac{r^{2}}{r_{0}^{2}}\right) \\
& T=T_{0}-\frac{q_{0}^{\prime \prime}}{k}\left(\frac{3}{4} r_{0}+\frac{r^{4}}{4 r_{0}^{3}}-\frac{r^{2}}{r_{0}}\right)
\end{aligned}
$$

The local entropy generation rate then follows from the cylindrical form of Equation (1) and is given by Equation (9).

$$
\dot{S}_{\text {gen }}^{\prime \prime \prime}=\frac{k\left\{\frac{q_{0}^{\prime \prime}}{k}\left(\frac{r^{3}}{r_{0}^{3}}-2 \frac{r}{r_{0}}\right)\right\}^{2}}{\left\{T_{0}-\frac{q_{0}^{\prime \prime}}{k}\left(\frac{3}{4} r_{0}+\frac{r^{4}}{4 r_{0}^{3}}-\frac{r^{2}}{r_{0}}\right)\right\}^{2}}+\frac{\mu\left(\frac{-4 V r}{r_{0}^{2}}\right)^{2}}{\left\{T_{0}-\frac{q_{0}^{\prime \prime}}{k}\left(\frac{3}{4} r_{0}+\frac{r^{4}}{4 r_{0}^{3}}-\frac{r^{2}}{r_{0}}\right)\right\}^{2}}
$$

The first and second terms on the right-hand side of the equation correspond to the entropy generation caused by the thermal gradients and viscous dissipation, respectively. 
The benchmark problem was modeled with TEMPEST in two-dimensional cylindrical coordinates with an axis of symmetry around the duct centerline. An axial duct length of $36 \mathrm{~m}$ was used to assure that fully developed flow was being modeled. Uniform inlet velocity and temperature profiles across the duct radius were assumed and the velocity and temperature profiles were allowed to develop along the axial length of the duct. Local entropy generation rates were computed at each of the radial nodes across the duct radius at the duct exit. The contribution to local entropy generation caused by thermal gradients and viscous dissipation were computed separately and then compared with the analytical solution. The wall temperature used in the analytical solution matched that used in the numerical simulation. A comparison of analytical and numerical local entropy generation rates across the duct radius is shown in Figure 1 .

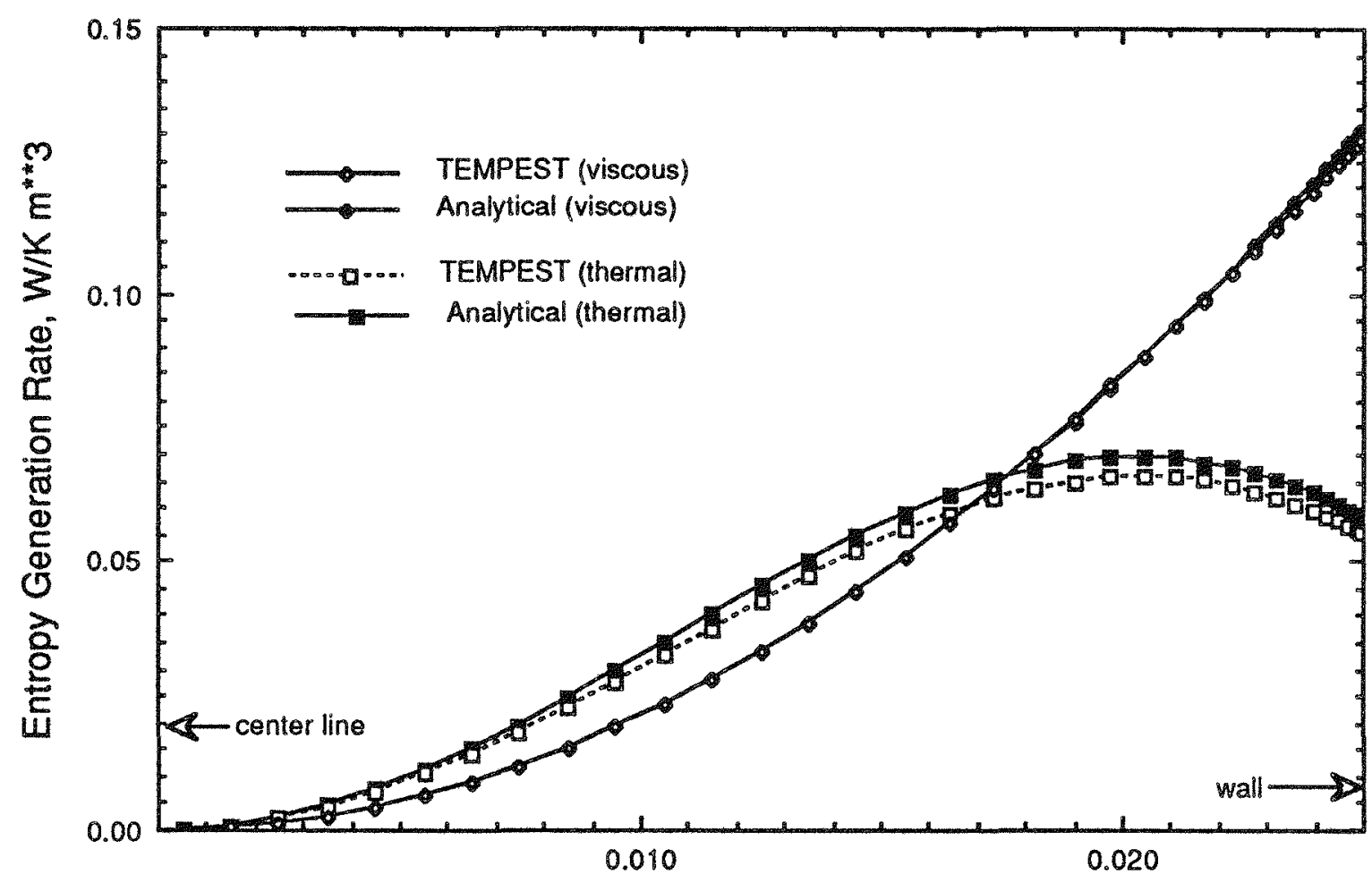

Radius, $m$

FIGURE 1. Entropy Generation for Laminar Flow with Constant Heat Flux 
The comparison demonstrates that both the TEMPEST code and the entropy generation subroutine function as expected for a laminar flow example. The $(\mathrm{k}-\mathrm{e})$ model used in TEMPEST has been thoroughly benchmarked for a variety of applications. The entropy subroutine was verified for turbulent flow by independent calculations of local entropy generation rates for established turbulent flow fields with convective heat transfer. 


\subsection{LOCAL ENTROPY GENERATION IN AN IMPINGING JET}

- The calculation of local entropy generation provides an analyst with theoretically valid information on the spatial and temporal distribution of thermodynamic losses in a thermal process and on the trade-offs associated with optimizing the process. The availability of this information can focus the analyst's attention on significant sources of irreversibilities and can provide the objective function for optimization. As part of this study, two problems were selected to demonstrate the calculation of local entropy generation in a complicated thermal process. The first problem consisted of calculating the local entropy generation in an impinging jet. Impingement heat transfer has been used for turbine blade cooling, cooling of electronic components, and for air heating in solar thermal power applications.

Understanding the structure of entropy generation in this process should be of direct use to heat transfer engineers. The second problem involves the evaluation of local entropy generation in the regenerator of a magnetic heat pump. The second problem is discussed in section 4.

\subsection{INTRODUCTION}

This problem consists of a jet of low-temperature fluid impinging on a heated wall and is shown schematically in Figure 2. The impinging jet problem involves viscous dissipation near the jet inlet and along the impinged wall. This results in entropy generation being concentrated in the same two regions. With surface heat transfer limited to the heated wall, the thermal entropy

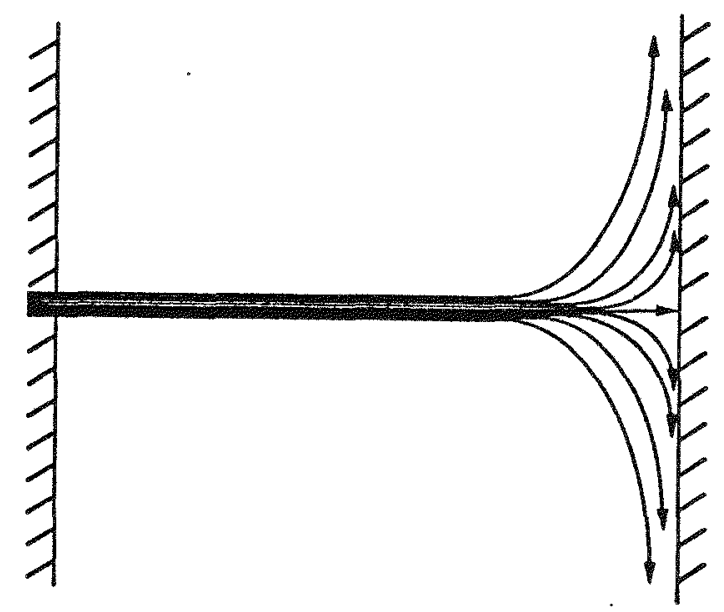

FIGURE 2. Impinging Jet

\section{1}


generation will primarily occur near the wall with turbulent mixing away from the wall producing local thermal gradients. Helium and glycerin were evaluated as heat transfer fluids. While helium is often used as a heat transfer fluid, glycerin was included as a limiting case where viscous dissipation becomes the dominant source of entropy generation, even at low velocities. Although TEMPEST and the associated entropy generation subroutine are fully transient and three-dimensional, the impinging jet was modeled as a two-dimensional slot jet operating at steady-state.

The physical arrangement and noding scheme used for the evaluation of the impinging jet are shown in Figure 3 . The fluid jet enters the computational domain in the lower corner of the left vertical boundary with a uniform specified velocity. In the horizontal direction the computational domain is bounded by two vertical walls; the left wall being an adiabatic, no slip surface, and the right wall being either an isothermal or constant heat flux, no slip surface. The upper horizontal surface is an unspecified outflow/inflow boundary for which the flow conditions are computed. Due to the symmetry of the jet, only one-half of the jet needs to be simulated. This means that the lower boundary will be a line of symmetry located on the centerline of the jet. Therefore, the lower horizontal boundary is modeled as having a rigid free-slip flow condition, with an adiabatic surface. The inlet jet has a width of $0.3 \mathrm{~m}$, whereas, the flow outlet covers the entire upper horizontal boundary with a width of $1.124 \mathrm{~m}$. The vertical dimension of the computational domain was arbitrarily limited to a $1.5 \mathrm{~m}$ length.

The computational noding scheme consisted of a $60 \times 40$ Cartesian grid with variable node spacing. The horizontal node spacing varied between $0.0711 \mathrm{~m}$ and $0.001 \mathrm{~m}$, while the vertical node spacing varied between $0.05 \mathrm{~m}$ and $0.01 \mathrm{~m}$. The high aspect ratio nodes were located near the vertical isothermal surface to capture the thermal and momentum boundary layers. Vertical node refinement was used to capture the shear layers of the jet as the jet entered the computational domain. All computations were performed on a Macintosh II (a) computer configured either with a MC68020 processor, or a

(a) Trademark of Apple Computers, Inc. Cupertino, California. 


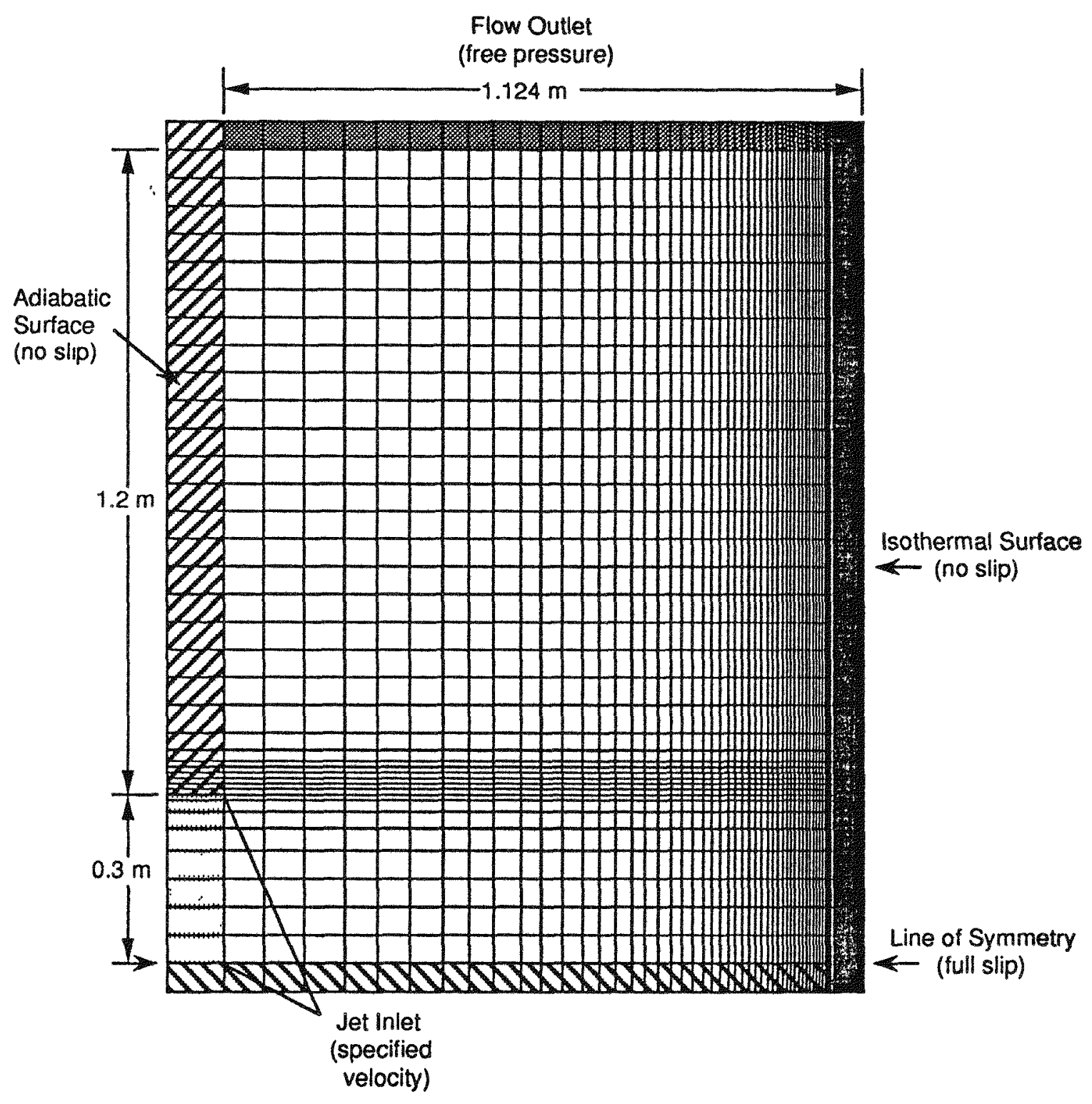

FIGURE 3. Computational Grid for the Impinging Jet Problem

MC68030 processor and MC68882 coprocessor. Typical central processing unit (CPU) times for the latter machine were around $14 \mathrm{~ms} /$ time-step/cell.

The jet impingement problem was simulated using thermal property data for glycerin and helium. One glycerin and four helium jet velocities were evaluated. Two thermal boundary conditions were considered for each fluid 
type and inlet jet velocity: 1) an isothermal surface $10^{\circ} \mathrm{C}$ above the inlet jet temperature and 2) a constant surface heat flux. viscosity, thermal conductivity, specific heat, and density were modeled as being independent of temperature. A total of 10 cases were simulated. The key parameters associated with the simulations are summarized in Table 1. The Reynolds number presented in Table 1 uses the diameter of the inlet jet as the characteristic length.

\subsection{RESULTS}

Results from the 10 simulations are presented in three forms in the following sections. First, the local entropy generation rate has been integrated over the complete computational domain for each of the simulations and the results have been tabulated. This is followed by a discussion of the structure of local entropy generation in several of the simulations. The last section presents the results of an error analysis.

The discussion of the results will focus on the insights afforded by computing and visualizing local entropy generation rates associated with an impinging jet. The graphical representations were created by a procedure that converts the color image into a pseudo gray-scale image through a

\section{TABLE 1. Simulation Parameters}

\begin{tabular}{|c|c|c|c|c|}
\hline No. & Fluid & Jet Reynolds No. & Prandt 1 No. & Thermal Boundary \\
\hline la & glycerin & $7.5 \times 10^{2}$ & 16610.0 & Isothermal \\
\hline $1 \mathrm{~b}$ & glycerin & $7.5 \times 10^{2}$ & 16610.0 & Heat flux, $1830.0 \mathrm{~W} / \mathrm{m}^{2}$ \\
\hline $2 a$ & helium & $3.0 \times 103$ & 0.556 & Isothermal \\
\hline $2 b$ & helium & $3.0 \times 10^{3}$ & 0.556 & Heat flux, $150.0 \mathrm{~W} / \mathrm{m}^{2}$ \\
\hline $3 a$ & helium & $3.0 \times 10^{4}$ & 0.556 & Isothermal \\
\hline $3 b$ & helium & $3.0 \times 10^{4}$ & 0.556 & Heat flux, $150.0 \mathrm{~W} / \mathrm{m}^{2}$ \\
\hline $4 a$ & helium & $1.5 \times 105$ & 0.556 & Isotherma 1 \\
\hline $4 b$ & helium & $1.5 \times 105$ & 0.556 & Heat flux, $150.0 \mathrm{~W} / \mathrm{m}^{2}$ \\
\hline $5 a$ & helium & $3.0 \times 105$ & 0.556 & Isothermal \\
\hline $5 b$ & helium & $3.0 \times 10^{5}$ & 0.556 & Heat flux, $150.0 \mathrm{~W} / \mathrm{m}^{2}$ \\
\hline
\end{tabular}


palette of interconnected symbols. Where relevant, the palette, with its associated scale, is shown below each figure.

\subsubsection{Integrated Entropy Generation}

The results of local entropy generation analysis can be used to optimize a device because the local entropy generation rate can be integrated over the complete domain to yield one objective function that combines the impact of different thermodynamic losses. In the case of the impinging jet, the integrated total entropy generation can be used to thermodynamically optimize the design (minimizing irreversibilities). In this evaluation, the impact of three design variables on thermodynamic performance was explored. First, the influence of the heated wall boundary conditions on the integrated total entropy generation was investigated. The second evaluation examined the impact of the jet velocity (or jet Reynolds number) on total integrated entropy generation. The final evaluation compared helium with glycerin as a heat transfer fluid for a impinging jet. In the first two cases, the results were able to provide information useful to an analyst. The third evaluation was unrealistic because glycerin would not be seriously considered as a heat transfer fluid, but the same procedure can be used as a criterion to choose between more realistic fluids. It should be noted that the integrated total entropy generation can only be obtained by conducting the evaluation of local entropy generation because analytical solutions do not exist for the velocity and temperature fields associated with a complicated process such as an impinging jet.

Table 2 summarizes the integrated entropy generation rates resulting from the 10 simulations. The second through the fourth columns in Table 2 present the local entropy generation rates integrated over the computational domain for entropy generation associated with viscous dissipation and thermal gradients, and total integrated entropy generation. The fifth column presents the average surface heat flux from the heated surface. For isothermal simulations, the local heat flux was a complex function of the vertical wall position, fluid properties, and jet Reynolds number, with the maximum heat fluxes occurring at the jet centerline. 
TABLE 2. Integrated Entropy Generation Rates

\begin{tabular}{|c|c|c|c|c|}
\hline No. & Viscous, W/K & Thermal, $\mathrm{W} / \mathrm{K}$ & Total, W/K & Heat Flux, W/m² \\
\hline $1 a$ & 0.233 & 0.145 & 0.377 & 1830 \\
\hline $1 b$ & 0.233 & 0.129 & 0.362 & 1830 \\
\hline $2 a$ & $0.551 \times 10^{-5}$ & $0.2397 \times 10^{-1}$ & $0.2397 \times 10^{-1}$ & 275 \\
\hline $2 \mathrm{~b}$ & $0.551 \times 10^{-5}$ & $0.637 \times 10^{-2}$ & $0.638 \times 10^{-2}$ & 150 \\
\hline $3 a$ & $0.105 \times 10-2$ & $0.865 \times 10^{-1}$ & $0.872 \times 10^{-1}$ & 944 \\
\hline $3 b$ & $0.105 \times 10^{-2}$ & $0.207 \times 10^{-2}$ & $0.313 \times 10^{-2}$ & 150 \\
\hline $4 a$ & 0.129 & 0.149 & 0.278 & 1840 \\
\hline $4 b$ & 0.129 & $0.998 \times 10^{-3}$ & 0.131 & 150 \\
\hline $5 a$ & 0.921 & 0.209 & 1.130 & 2740 \\
\hline $5 b$ & 0.921 & $0.644 \times 10^{-3}$ & 0.933 & 150 \\
\hline
\end{tabular}

A review of the results demonstrates the importance of local entropy generation calculations. There are two significant findings.

- optimum jet Reynolds number - One of the objectives of the study was to determine the optimum jet Reynolds number. It was anticipated that the entropy generation caused by viscous dissipation would increase with the jet Reynolds number, while the entropy generation caused by thermal gradients would decrease, resulting in an optimum Reynolds number. A comparison of simulations $2 b, 3 b, 4 b$, and $5 b$ show this to be the case. As expected, the integrated entropy generation caused by viscous dissipation increases with increasing Reynolds number, while the integrated entropy generation caused by thermal gradients decreases. The minimum integrated total entropy generation occurs at a Reynolds number of approximately $3.0 \times 10^{4}$. This suggests that if the designer of an impinging jet heat exchanger wants to minimize thermodynamic losses in a heat exchanger with the characteristics of the sample problem, the device should be designed to operate with a jet Reynolds number of around $3.0 \times 10^{4}$.

- heat transfer fluid selection - Simulation 1a and $4 a$ were used to compare helium with glycerin as a heat transfer fluid because the integrated heat flux is similar for both simulations. The results show that the total entropy generation associated with using glycerin as the heat transfer fluid is significantly larger than the entropy generation rate associated with using helium. As expected, the results suggest that glycerin is not an attractive candidate for use in impingement heat exchangers. While a rigorous comparison of heat transfer fluids was beyond the scope of this study, the use of integrated total entropy generation can provide a method of comparing alternative fluids. 
Other design variables, such as the spacing between the jet and the heated wall and the angle of incidence for the jet, could also be optimized using local entropy generation analysis, but the limited scope of this demonstration prevented further investigation.

\subsubsection{Local Entropy Generation}

The local entropy generation results are presented for two simulations. The first three figures show the components of local entropy generation and total entropy generation for case 3a (a helium jet with a jet Reynolds number of $3.0 \times 10^{4}$ ). The last two figures show entropy generation caused by viscous dissipation and thermal gradients for case 1 a (a glycerin jet with a jet Reynolds number of $7.5 \times 10^{2}$ ).

Figures 4, 5, and 6 depict fields of local entropy generation rates for simulation $3 a$, separated into the viscous dissipation component, thermal gradient component, and total entropy generation rate. The three figures are equivalently scaled using the natural logarithm of the entropy generation. The logarithmic scale ranges from $4.63 \times 10^{-9} \mathrm{~W} / \mathrm{K} \mathrm{m} \mathrm{m}^{3}$ to $228.2 \mathrm{~W} / \mathrm{K} \mathrm{m}^{3}$. The local entropy generation caused by viscous dissipation is shown in Figure 4 . The results show that local entropy generation caused by viscous dissipation is concentrated in the shear layers near the jet inlet and the vertical wall boundary layers. Without logarithmic scaling, the details in the figure are lost, with the perceivable entropy generation collapsing to a thin shear and boundary layer. Local entropy generation caused by thermal gradients is shown in Figure 5, where it appears as an enlarged boundary layer along the heated vertical surface. The relatively high entropy generation rates that occur within the entrance shear layers of the jet result from the recirculating helium at elevated temperatures mixing with the cooler inlet jet fluid. The spatial distribution of total local entropy generation is presented in Figure 6 and shows that entropy generation caused by viscous dissipation dominates in the jet entrance shear region, while the thermal gradient component of local entropy generation dominates in the boundary layer along the vertical, heated wall. As one would expect, the observed distribution is strongly dependent on the flow structure and thermal boundary conditions. 


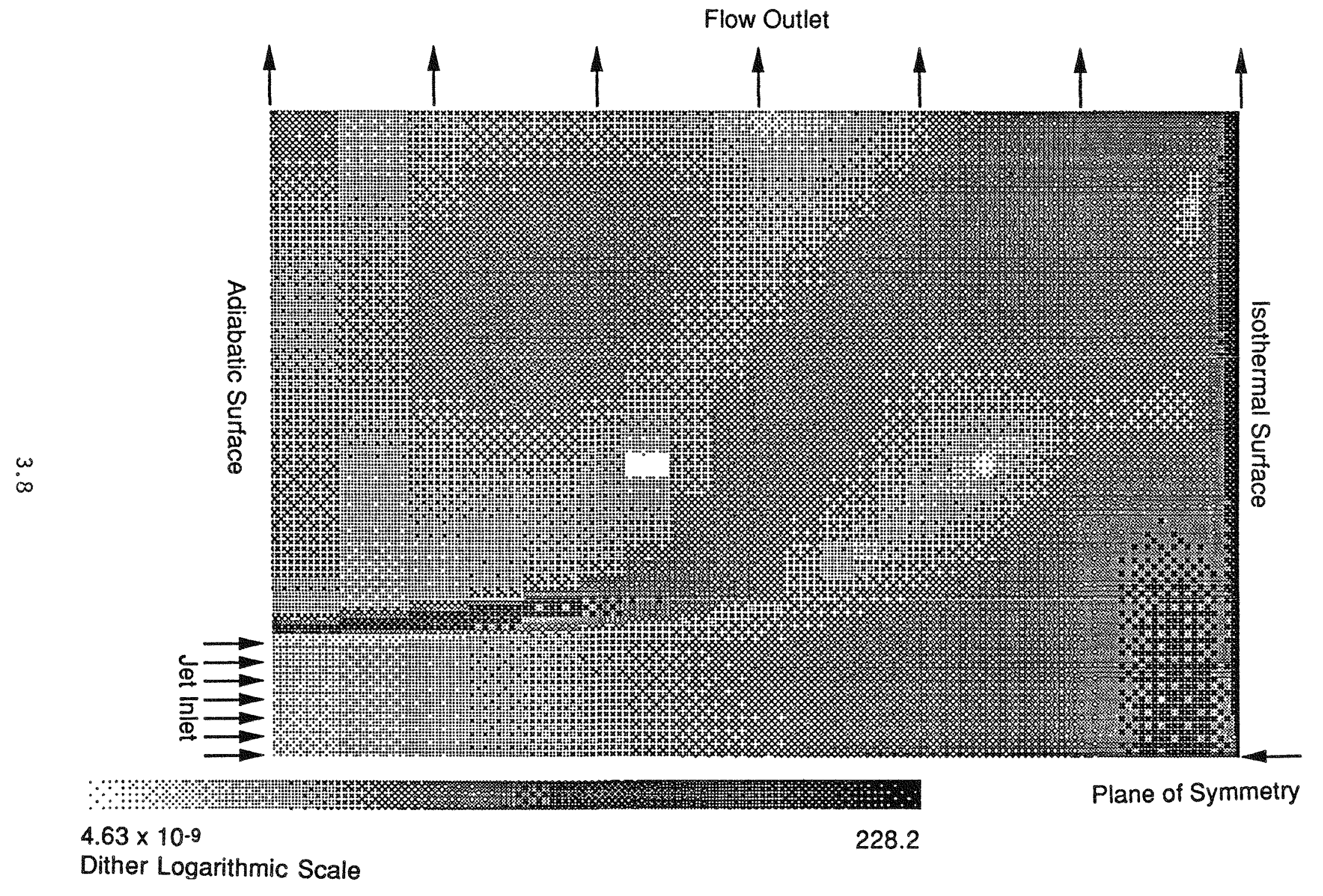

FIGURE 4. Viscous Component of Local Entropy Generation $W / \mathrm{m}^{3} \mathrm{~K}$ 


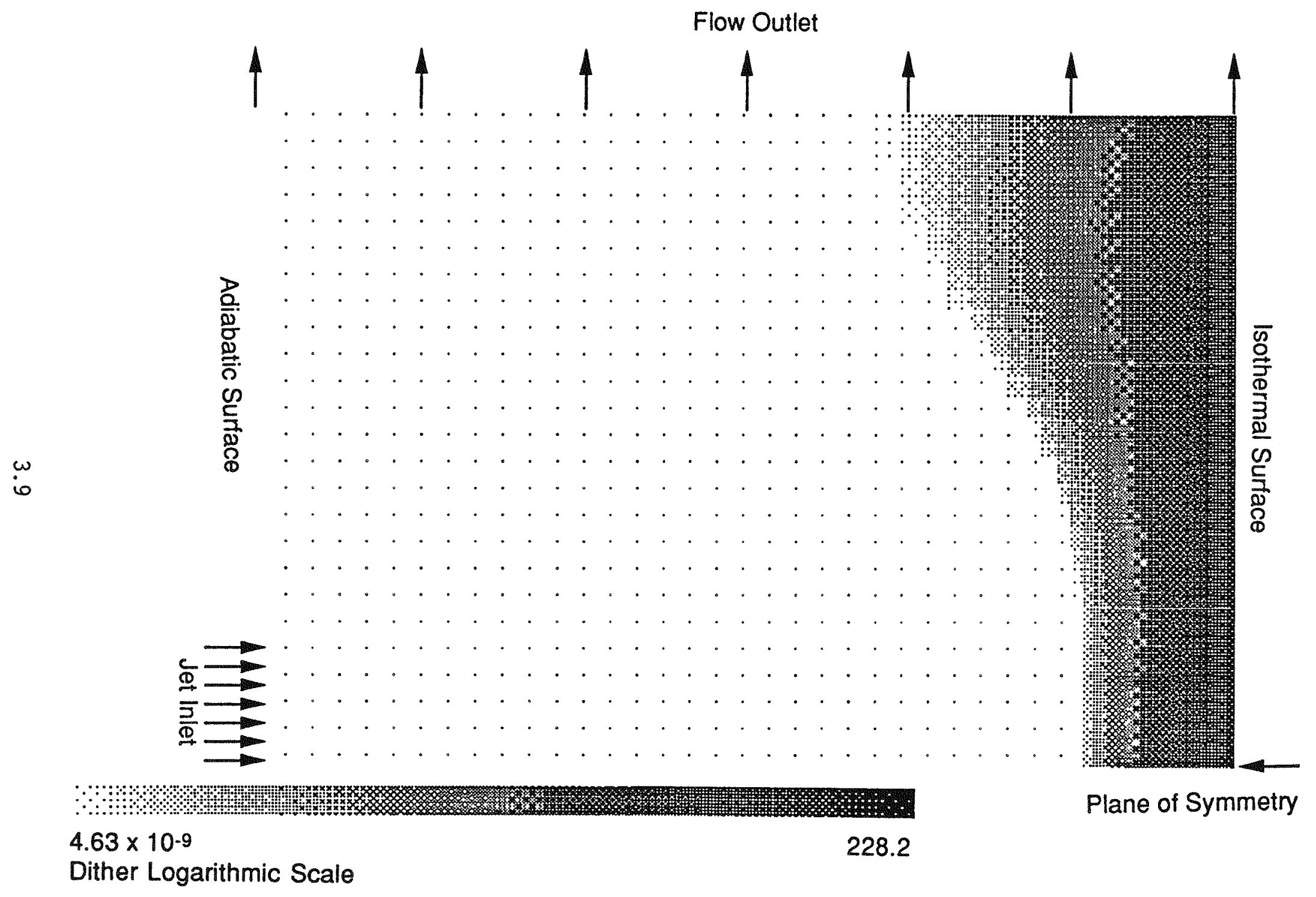

FIGURE 5. Thermal Component of Local Entropy Generation $\mathrm{W} / \mathrm{m}^{3} \mathrm{~K}$ 


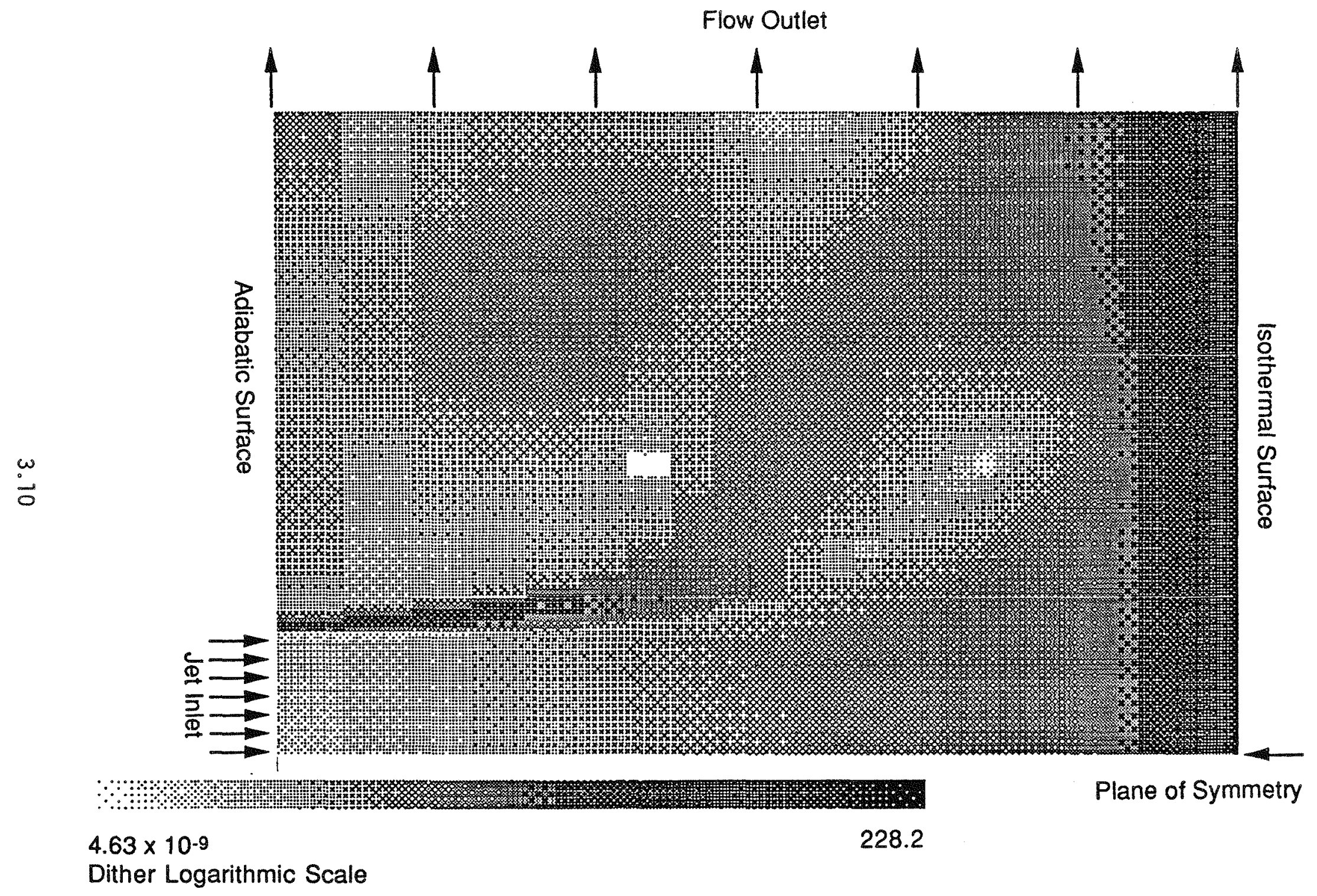

FIGURE 6. Total Local Entropy Generation $W / \mathrm{m}^{3} \mathrm{~K}$ 
The impinging jet was also simulated using glycerin as the heat transfer fluid. The components of local entropy generation for a glycerin jet impinging on an isothermal heated surface (simulation 1a) are depicted in Figures 7 and 8 . Figures 7 and 8 are scaled by the natural logarithm of entropy generation rates. The viscous dissipation component of local entropy generation is shown in Figure 7 and it is quite different from its counterpart for the helium jet. A significant amount of entropy generation occurs in the glycerin jet as it decelerates upon entering the computational domain. Moreover, a more equal distribution of viscous dissipation is occurring along the adiabatic and heated vertical surfaces. This is in contrast to the helium jet simulation where the viscous dissipation was confined to the entrance shear region and the impingement wall boundary layer. These differences in the viscous dissipation fields are attributed to the differences between jet Reynolds numbers and fluid physical properties.

The results of the local entropy generation analysis are important because they show the structure of entropy generation. When helium is the jet fluid, the results show that entropy generation is concentrated in the boundary layer and that the analyst can understand the distribution of entropy generation by understanding the behavior of the boundary layer. When glycerin is used, entropy generation in the shear layer becomes much more important.

Local entropy generation has an additional application because it can be viewed as the fundamental source of cost generation in a process. While this issue was beyond the scope of the current study, in theory, it should be possible to assign a cost to each component of entropy generation. It would then be possible to produce figures similar to Figures 4 through 8 that show the spatial distribution of cost generation.

\subsubsection{Error Analysis}

An estimate of the relationship between errors in the primitive variables of temperature and velocity, and errors in the entropy generation components 


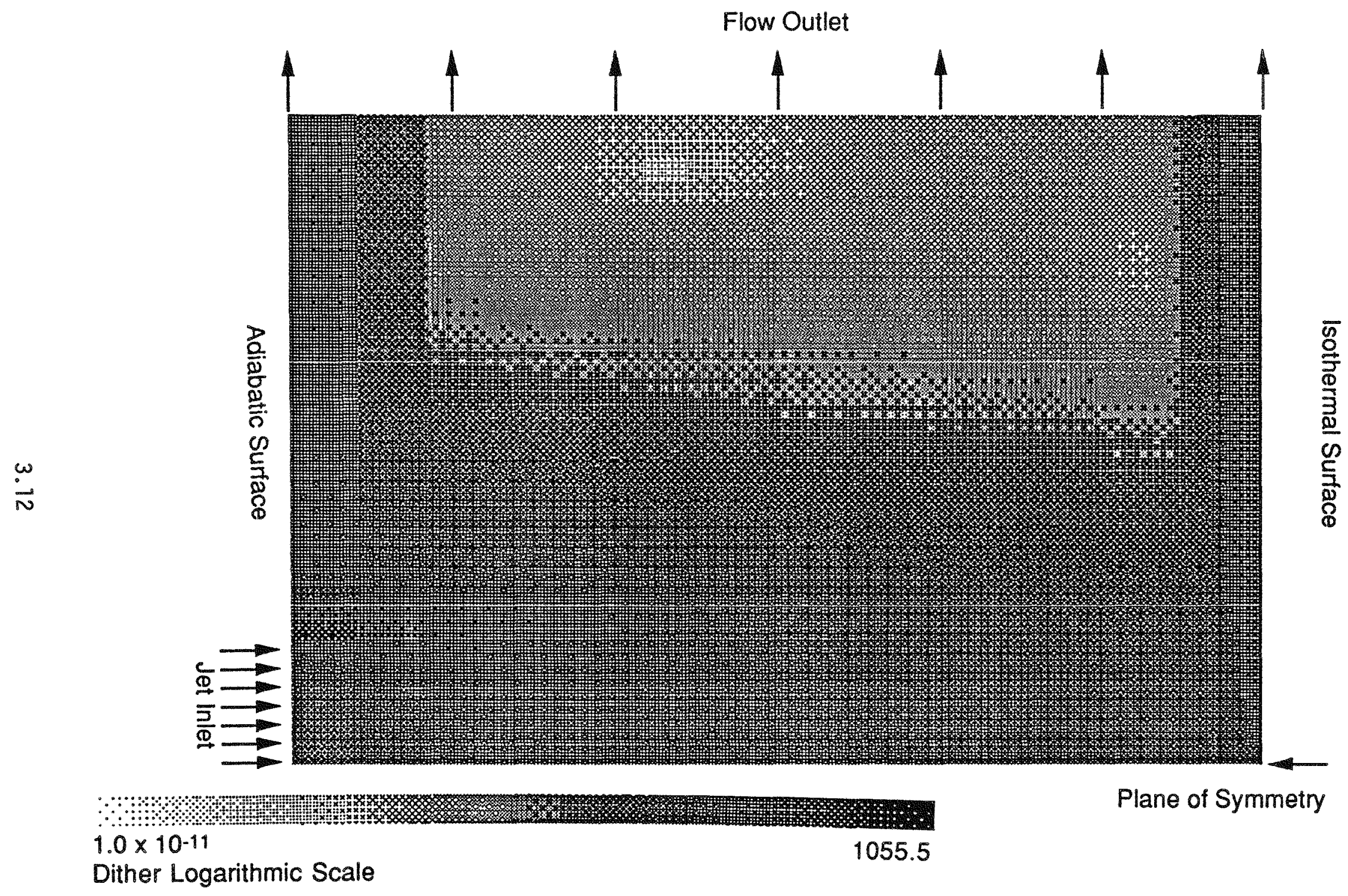

FIGURE 7. Viscous Component of Local Entropy Generation $\mathrm{W} / \mathrm{m}^{3} \mathrm{~K}$ 


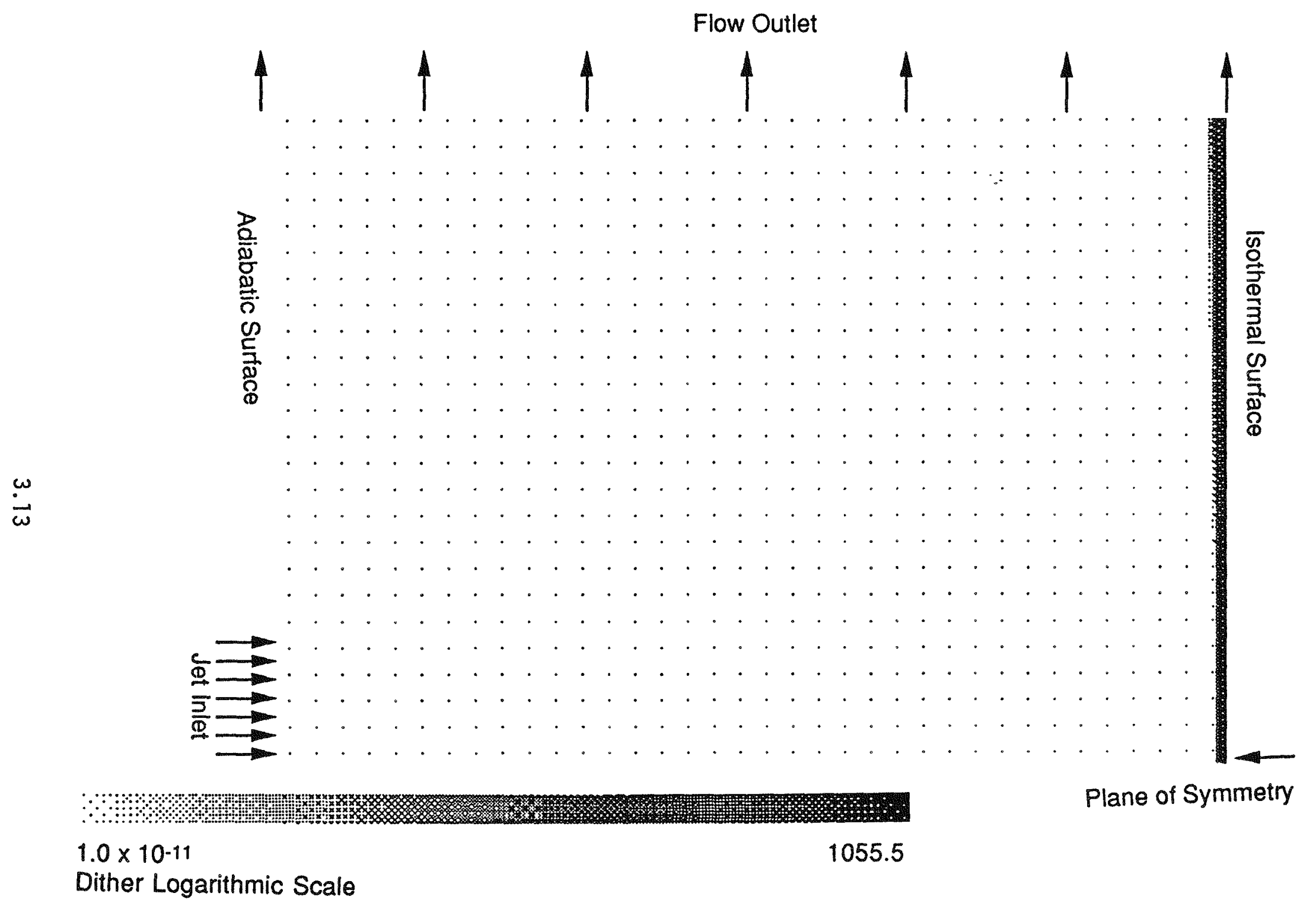

FIGURE 8. Thermal Component of Local Entropy Generation $W / m^{3} \mathrm{~K}$ 
may be made with a few assumptions. For the viscous dissipation component, it is assumed that:

$$
\frac{\partial u}{\partial x}=\frac{\partial u}{\partial y}=\frac{\partial v}{\partial x}=\frac{\partial v}{\partial y} ; \text { and } \epsilon_{u}=\epsilon_{v} ;
$$

Then, an estimate of the error in the viscous component of entropy generation that corresponds to a level error in the velocity field may be expressed as the following, for a first-order finite difference scheme:

$$
\epsilon_{\mathrm{sgv}}=32 \frac{\mu}{T}\left\{\frac{\epsilon_{v}}{\Delta x} \frac{\Delta u}{\Delta x}+\frac{\epsilon_{v}^{2}}{\Delta x^{2}}\right\}
$$

If the velocity gradient $\Delta u / \Delta x$ is approximated by the gradient across the turbulent momentum boundary layer on the heated surface at the volume exit, $\Delta x$ is approximated by the average node spacing, and the second term of Equation (9) is ignored, a relation between an error in velocity and the viscous component of entropy generation may be obtained. These relations are summarized in Table 3 for the helium simulations.

A similar analysis was performed for an error in the thermal component of entropy generation with dependence on the error in the temperature field. For the thermal error analysis, it was assumed that

$$
\frac{\partial T}{\partial x}=\frac{\partial T}{\partial y} ;
$$

TABLE 3. Relation Between Primitive Errors and Entropy Errors

Jet Reynolds No.

$3.0 \times 10^{3}$

$3.0 \times 10^{4}$

$1.5 \times 10^{4}$

$3.0 \times 105$

\begin{tabular}{l} 
Viscous Relation \\
\hline$\epsilon_{\mathrm{sgv}}=2.97 \times 10^{-4} \epsilon_{\mathrm{u}}$ \\
$\epsilon_{\mathrm{sgv}}=4.74 \times 10^{-3} \epsilon_{\mathrm{u}}$ \\
$\epsilon_{\mathrm{sgv}}=3.28 \times 10^{-2} \epsilon_{\mathrm{u}}$ \\
$\epsilon_{\mathrm{sgv}}=7.54 \times 10^{-2} \epsilon_{\mathrm{u}}$
\end{tabular}

Thermal Relation $\epsilon_{\mathrm{Sgt}}=5.05 \times 10^{-2} \epsilon_{\mathrm{T}}$ $\epsilon_{\mathrm{Sgt}}=8.77 \times 10^{-2} \epsilon_{\mathrm{T}}$ $\epsilon_{\mathrm{Sgt}}=1.21 \times 10^{-1} \epsilon_{\mathrm{T}}$ $\epsilon_{\mathrm{Sgt}}=1.40 \times 10^{-1} \epsilon_{\mathrm{T}}$ 
The thermal gradient $\Delta T / \Delta x$ was approximated by the gradient across the thermal boundary layer on the heated surface at the volume exit; $\Delta x$ was approximated by the average node spacing; and the analogous term to the second term of Equation (9) was ignored. The relations between an error in temperature and the thermal component of entropy generation are also summarized in Table 3 for the helium simulations.

In all cases the error resulting from the first-order finite difference scheme is small compared to the calculated entropy generation. 


\subsection{LOCAL ENTROPY GENERATION IN A MAGNETIC HEAT PUMP REGENERATOR}

The second demonstration of local entropy generation analysis consists of an evaluation of local entropy generation in the regenerator of a magnetic heat pump. Barclay (1987) and Kirol et al. (1984) have shown that the magnetic heat pump has attractive thermodynamic performance but its performance is strongly influenced by the effectiveness of the regenerator. The work reported in this section uses local entropy generation analys is to evaluate a regenerator design proposed by Kirol et al. (1984) for a rotary magnetic heat pump.

\subsection{INTRODUCTION}

A magnetic heat pump uses the entropy changes associated with the magnetization and demagnetization of a magnetic material to transfer thermal energy from a low-temperature reservoir to a high-temperature reservoir. A number of heat pump configurations have been investigated, but Kirol et al. (1984) concluded that a rotary design appears to be most attractive. In the rotary heat pump design, thin ring-shaped sheets of magnetic material are rotated through fixed magnetic fields. A schematic arrangement of the magnetic heat pump is shown in Figure 9. A regenerator fluid (such as water) is pumped in the direction opposite to the rotation of the magnetic material because efficient operation of the heat pump requires regeneration. When the material enters a magnetic field (state point 2 on Figure 9), the temperature of the material increases and a fraction of the thermal energy is extracted ("heat to high-temperature sink" on Figure 9); the balance is used to heat the regenerator fluid (process occurring from state point 2 to 3 on Figure 9 ). When the material is demagnetized, its temperature drops (state point 3 ) and heat is added to the magnetic material from both the low-temperature reservoir ("heat from low-temperature source") and the regenerator fluid (process occurring from state point 4 to 1). A more detailed description of the concept is presented in Kirol et al. (1984). 
Regeneration occurs at two locations in the cycle: between state points 2 and 3 , hot magnetic material is used to heat regenerator fluid while the regenerator fluid is used to heat the magnetic material between state points 4 and 1. Studies of the magnetic heat pump concept show that an efficient design must have a high-performance regenerator. This has led researchers to apply advanced analytical techniques, such as second law analysis, to the evaluation of the regenerator. Several researchers have used a lumped parameter second law analys is to investigate a magnetic heat pump regenerator (Kirol et a1. 1984; Barclay 1984; Barclay and Sarangi 1984) and the results have been used to identify optimum regenerator characteristics, resulting in a design that minimizes second law losses.

The research reported in this section involves the application of local entropy generation analysis to the rotary magnetic heat pump regenerator design described in Kirol et a1. (1984). Local entropy generation analysis determines the local generation of entropy in solids and convecting fluids based on the temperature and flow fields. This approach allows the explicit investigation of trade-offs between sources of entropy generation and design optimization (White and Drost 1989).

\subsection{METHODOLOGY}

The analysis of the magnetic heat pump regenerator consisted of three evaluations. First, local entropy generation in a typical flow path in the regenerator was evaluated using the TEMPEST CFD code to predict velocity and temperature fields. Local entropy generation was then integrated over the flow path, resulting in an estimate of total entropy generation for that specific regenerator design. Subsequently, integrated local entropy generation was used as the figure-of-merit for parametric evaluations of design variations. The second evaluation concentrated on investigating the use of flow disrupters for heat transfer enhancement. In this case, local entropy generation was evaluated for a small section of the regenerator including several flow disrupters. The final evaluation used the results of the flow disrupter analysis to test a widely used figure-of-merit for the thermodynamic evaluation of heat transfer enhancement. 

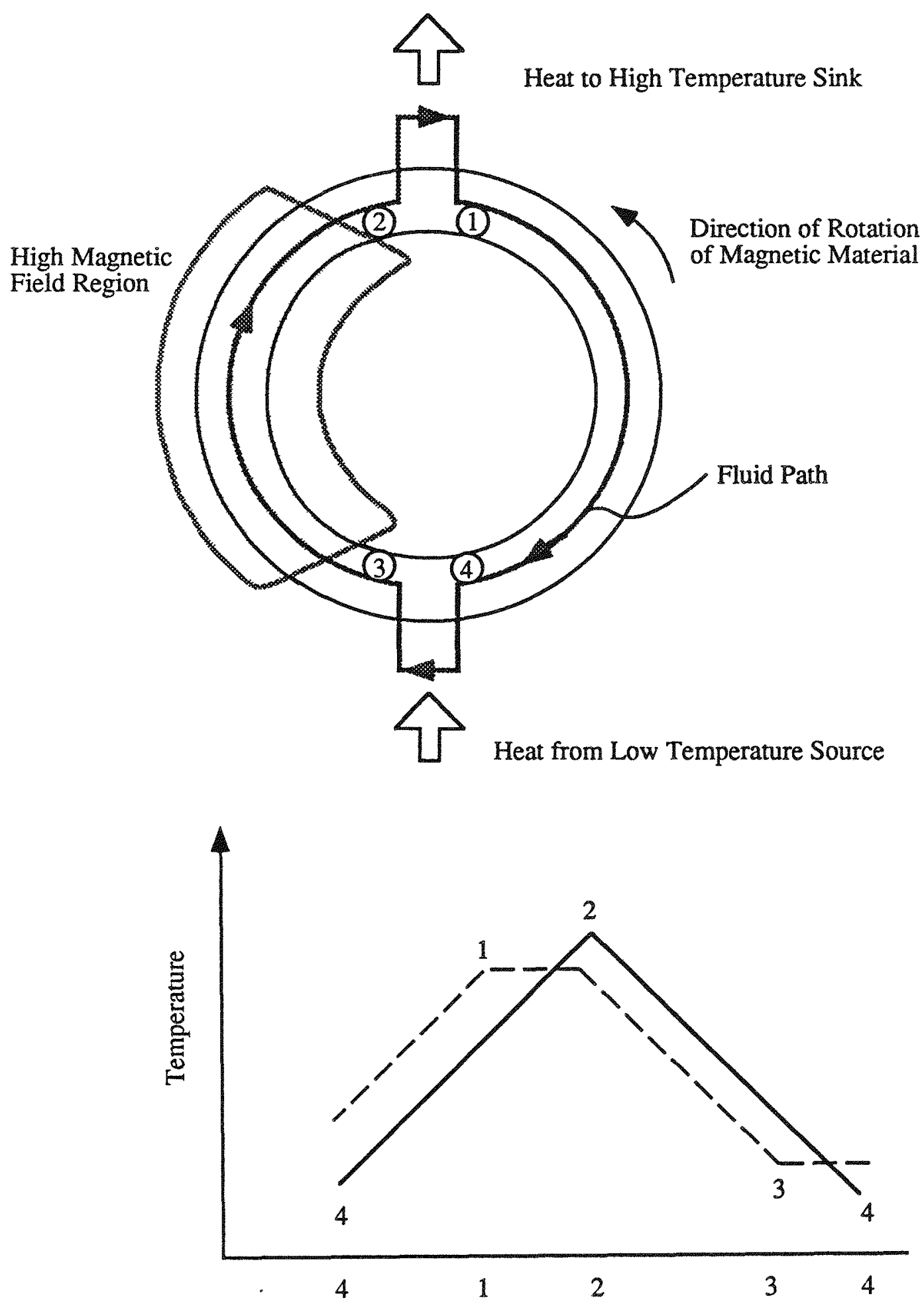

State Point

FIGURE 9. Rotary Magnetic Heat Pump (Kirol et al. 1984) 
While this study focused on the minimization of thermodynamic losses rather than using an economic figure-of-merit, local entropy generation is also the source of local cost generation. If the cost of the various sources of entropy generation was available, it is possible to determine local cost generation and the optimum regenerator configuration based on minimum cost generation.

\subsubsection{Parametric Analysis}

The parametric analysis was conducted by calculating integrated local entropy generation for a typical flow path in the regenerator during one phase of the regeneration cycle during which heat is transferred from the hot magnetic material to the regenerator fluid. The regenerator flow path model is shown in Figure 10. The regenerator flow path consists of two sheets of magnetic material separated by the regenerator fluid. Hot magnetic material enters the regenerator and is assumed to be moving to the left. Cool regenerator fluid enters the regenerator and moves to the right while thermal energy is transferred from the magnetic material to the regenerator fluid. The incoming regenerator fluid was assumed to have a constant inlet velocity profile. Figure 10 shows the fully developed velocity profile that would be obtained as the regenerator fluid flows through the regenerator.

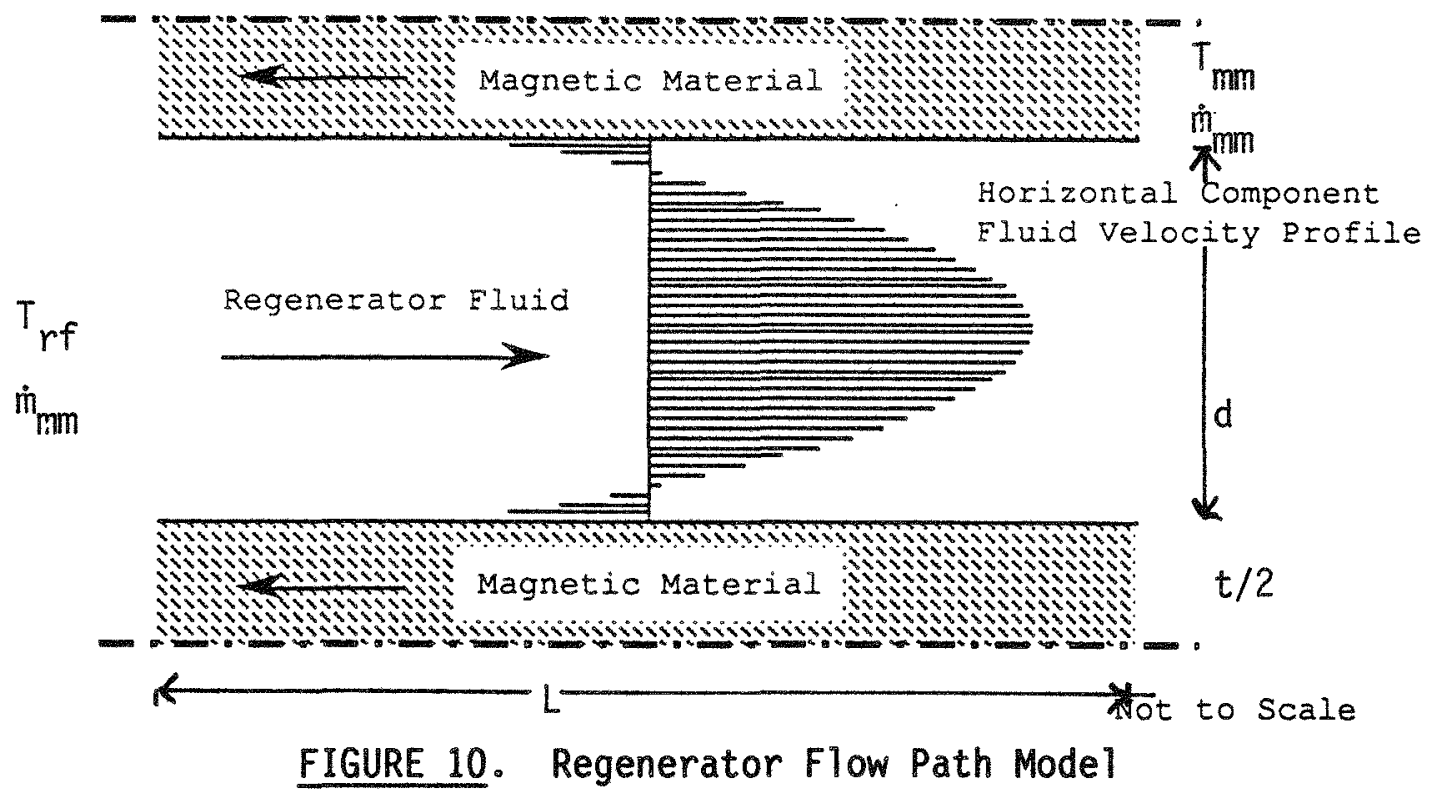


The regeneration process involves four thermodynamic losses: 1) entropy generation caused by heat transfer across the finite temperature difference between the magnetic material and the regenerator fluid [given by Equation (2) applied to the regenerator fluid], 2) entropy generation caused by conduction of thermal energy along the magnetic material [given by Equation (2) applied to the magnetic material], 3) entropy generation caused by viscous dissipation in the regenerator fluid [given by Equation (3) applied to the regenerator fluid], and 4) residual losses (losses associated with the incomplete cooling of the magnetic material).

The fourth loss mechanism assigns a penalty for a regenerator design that does not extract all of the available thermal energy in the magnetic material. This loss can also be viewed as a source of entropy generation if we consider the fate of the thermal energy remaining in the magnetic material after it leaves the regenerator. The entropy generation can be calculated if it is assumed that the flow of the heat source fluid is sufficiently high to cool the magnetic material to the temperature of the regenerator fluid as it enters the regenerator, in which case the entropy generation associated with residual losses is given by Equation (13).

$$
\dot{S}_{\text {Lostw }}=\frac{m C p}{T_{\text {ref }}}\left(T-T_{\text {ref }}\right) \dot{m} C p \ln \frac{T}{T_{\text {ref }}}
$$

Parametric investigations were conducted by selecting a set of design parameters and using the TEMPEST CFD code to determine the temperature and velocity profiles in the regenerator fluid, the temperature field in the magnetic material, the exiting temperature of the magnetic material, and the local entropy generation. The regenerator was a particularly attractive application for the numerical evaluation of local entropy generation because most simulations involved laminar flow conditions. This eliminated concerns about appropriate turbulence modeling and modeling turbulence generation near solid boundaries. Some flow disrupter simulations probably involved turbulent flow, but we assumed laminar flow for all cases because this assumption would result in a conservative estimate for heat transfer enhancement. Therefore, all cases were modeled as being laminar. 
The total entropy generation for a specific design was determined by integrating the local entropy generation over the magnetic material and regenerator fluid and then adding it to the entropy generation associated with remnant losses. This resulted in a figure-of-merit that included the impacts of all four relevant thermodynamic loss mechanisms described above.

The base case regenerator geometry and bulk flow characteristics were taken from the optimum design, as identified by Kirol et al. (1984). $\mathrm{Y}_{2}\left(\mathrm{Fe}_{x} \mathrm{Co} 1-\mathrm{x}\right) 17$ was used for the magnetic material and water was assumed to be the regenerator fluid. Material properties for the magnetic material are presented in Kirol et a1. (1984). Key base case parameters are defined on Figure 10 and are presented below.

- regenerator length $(L)=0.28 \mathrm{~m}$

- magnetic material thickness $(t)=2.6 \times 10^{-4} \mathrm{~m}$

- flow path width (d) $=2.6 \times 10^{-4} \mathrm{~m}$

- magnetic material inlet temperature $\left(T_{\mathrm{mm}}\right)=160^{\circ} \mathrm{C}$

- regenerator fluid inlet temperature $(\operatorname{Trf})=104^{\circ} \mathrm{C}$

- magnetic material mass flow rate $\left(\dot{\mathrm{m}}_{\mathrm{mm}}\right)=1.466 \mathrm{~kg} / \mathrm{s}(\mathrm{m})$

- regenerator mass flow rate $\left(\dot{m}_{r f}\right)=0.1992 \mathrm{~kg} / \mathrm{s}(\mathrm{m})$

The parametric study investigated the impact of three design parameters on regenerator performance.

- Relative velocity between the regenerator fluid and the magnetic material - Entropy generation and regenerator capacity will be a strong function of the relative velocity between the fluid and the magnetic working material. Usually, entropy generation and the capacity of the regenerator will increase monotonically with velocity because increased velocity will result in increased heat transfer (and hence an increased entropy generation).

- Length of the flow path - Entropy generation can be determined as a function of the length of a flow path. As the length of the flow path increases, heat transfer entropy generation and remnant losses will decrease, while viscous losses will increase. Consequently, there will be one regenerator length that results in minimum entropy generation.

- Ratio of thermal capacity - The regenerator can be viewed as a counterflow heat exchanger. As with any counterflow heat exchanger, the ratio of the thermal capacities (the product of mass flow rate and 
specific heat) of the two fluids will affect entropy generation. Based on earlier evaluations of entropy generation in counterflow heat

exchangers, it was anticipated that balanced flow would result in minimum entropy generation (Bejan 1982).

\subsubsection{Evaluation of the Flow Disrupter Concept}

The results of parametric evaluations showed that the base case design for the regenerator was not the optimum based on thermodynamic performance. The thermal losses were several orders of magnitude larger than the viscous losses. This suggested that the design should include enhancement techniques that improve heat transfer, even at the expense of a substantial increase in viscous dissipation losses. The inclusion of enhancement techniques is difficult because the magnetic material moves past seals as it rotates inside the heat pump casing. The seals prevent the use of typical enhancement techniques, such as surface roughening. An alternative approach is to use a stationary flow disrupter located between the plates of magnetic material and attached to the casing. In this case, both the magnetic material plates and the regenerator fluid move past the stationary flow disrupter. The flow disrupter is shown in Figure 11.

Optimization of the flow disrupter design involved the trade-off of several sources of entropy generation. Local entropy generation analysis was used to evaluate the sources and distribution of entropy generation associated with the disrupter. It was anticipated that a number of flow disrupters would

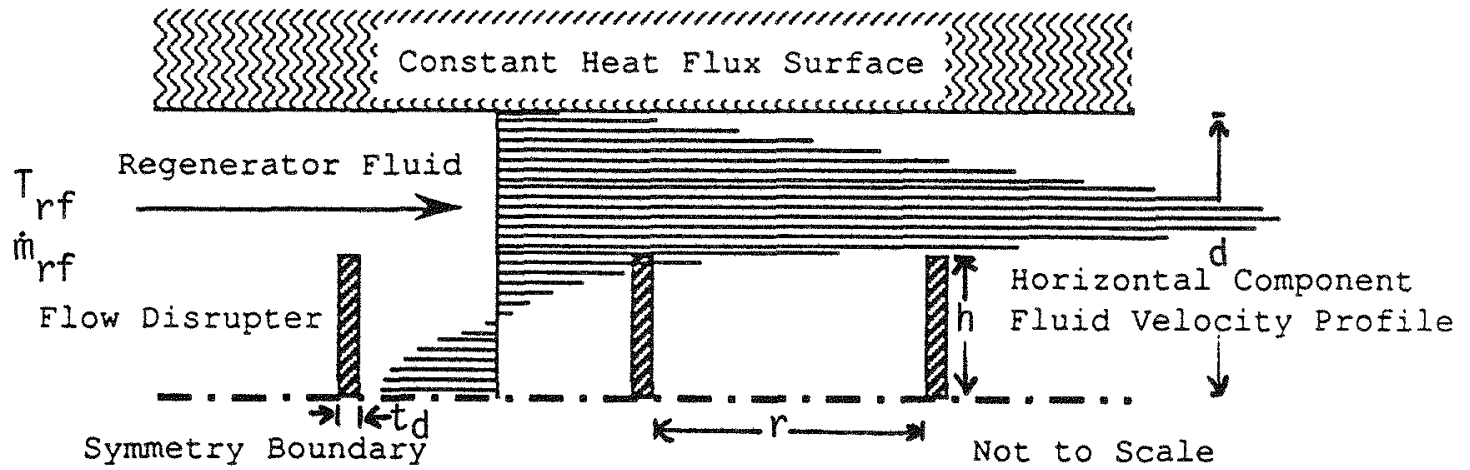

FIGURE 11. Flow Disrupter 
be located along the regenerator but a tractable simulation required that only a small segment of the regenerator be evaluated. Figure 11 shows the model used for numerical simulation. The magnetic material was not included in the simulation; instead a constant heat flux boundary condition was imposed, with the heat flux being representative of the heat fluxes encountered in the parametric studies described above. The inlet velocity was the fully developed velocity profile obtained from a numerical simulation of an infinite series of flow disrupters. As shown in Figure 11, three disrupters were included in the simulation. The major design variable included in the study was disrupter height. The impact of disrupter spacing or shape on the device's thermodynamic performance could also be investigated but these evaluations were beyond the scope of the present study. The geometric and flow characteristics of the disrupter model include:

- flow path width (d) $=2.6 \times 10^{-4} \mathrm{~m}$

- disrupter thickness $\left(t_{d}\right)=5 \mu m$

- disrupter spacing $(R)=150 \mu \mathrm{m}$

- disrupter height $(H)=$ variable

- inlet water mass flow rate $\left(\dot{\mathrm{m}}_{\mathrm{rf}}\right)=75 \mathrm{gm} / \mathrm{s}(\mathrm{m})$

- inlet water temperature $\left(T_{r f}\right)=130^{\circ} \mathrm{C}$

- wall heat flux $\left(\dot{q}_{0}^{\prime \prime}\right)=6.675 \times 10^{5} \mathrm{~W} / \mathrm{m}^{2}$

\subsubsection{Evaluation of Heat Exchanger Thermodynamic Figures-of-Merit}

The final evaluation used the results of the flow disrupter study to compare two figures-of-merit for heat transfer enhancement. The first figureof-merit proposed by Bejan was based on entropy generation. The second figureof-merit is a traditional first law approach to evaluating heat exchanger enhancement.

\subsection{RESULTS}

The results of the parametric analysis are included in Section 4.3.1 followed by a discussion of the evaluation of the flow disrupter concept presented in Section 4.3.2. 


\subsubsection{Parametric Analysis of Base Case Design}

The parametric evaluations consisted of three sensitivity studies. Table 4 presents the results of varying the length of the regenerator while holding inlet temperatures, ratio of $\dot{\mathrm{m}} \mathrm{Cp}$, and magnetic material and regenerator fluid velocities constant.

A review of Table 4 shows several interesting trends. First, the base case design is far from optimum. The optimum regenerator length, based on minimum entropy generation, is approximately $3.8 \mathrm{~m}$ as compared to the $0.28 \mathrm{~m}$ length used in the base case. This does not necessarily imply that the base case design is a poor selection because the use of a longer regenerator would substantially increase the size (and cost) of the magnetic heat pump. However, significant improvements in performance could be obtained by lengthening the regenerator.

Assuming that the physical dimensions of the regenerator are limited to the base case length of $0.28 \mathrm{~m}$, the results show that the viscous losses are approximately $1 \%$ of the thermal losses. This suggests that enhancements that reduce heat transfer related entropy generation should be considered even if they result in a substantial increase in viscous losses. As an example, an enhancement technique that reduces heat transfer losses by $10 \%$ could cause a $1000 \%$ increase in viscous losses and still result in improved thermodynamic performance. The low viscous losses also indicate that the designer need not

TABLE 4. Integrated Entropy Generation as a Function of Regenerator Length

\begin{tabular}{|c|c|c|c|c|c|c|c|c|}
\hline Case & $\begin{array}{l}\text { Regenerator } \\
\text { Length, } \mathrm{m}\end{array}$ & C & $\operatorname{Re}$ & $\begin{array}{l}S_{\text {Si is }} \\
W / K \\
\end{array}$ & $\begin{array}{l}\text { STham, } \\
\text { SW/K } \\
\end{array}$ & $\begin{array}{l}\dot{S}_{\text {Thrfo }} \\
\text { W/K } \\
\end{array}$ & $\begin{array}{l}\dot{S}_{\text {Lostw }} \\
W / K \\
\end{array}$ & $\begin{array}{c}\dot{S}_{\text {Total }} \\
\text { W/K } \\
\end{array}$ \\
\hline 1 (Base) & 0.28 & 1 & 915 & 0.011 & 0.394 & 0.958 & 0.158 & 1.51 \\
\hline 2 & 1.40 & 1 & 915 & 0.0539 & 0.1040 & 0.250 & 0.024 & 0.431 \\
\hline 3 & 2.80 & & 915 & 0.108 & 0.0541 & 0.130 & 0.023 & 0.315 \\
\hline 4 & 3.80 & 1 & 915 & 0.147 & 0.0410 & 0.0981 & 0.008 & 0.294 \\
\hline 5 & 8.80 & 1 & 915 & 0.337 & 0.0197 & 0.0472 & 0.003 & 0.407 \\
\hline 6 & 0.028 & 1 & 915 & 0.001 & 0.728 & 1.66 & 1.85 & 4.24 \\
\hline
\end{tabular}


consider techniques for reducing viscosity related losses. An example would be the use of viscosity reducing additives. The benefit of using an additive would be insignificantly small compared to the thermal losses.

Finally, the results show that entropy generation caused by conduction in the magnetic material is a significant fraction of the total losses. Kirol et a1. (1984) concluded that conduction losses could be ignored. Our results suggest that this is not the case. Conduction entropy generation contributes between $5 \%$ and $25 \%$ of the total entropy generation.

Table 5 presents the results of varying $C$, the ratio of thermal capacities $\left(\dot{\mathrm{m}}_{\mathrm{mm}} \mathrm{C}_{\mathrm{mm}} / \dot{\mathrm{m}}_{\mathrm{rf}} \mathrm{C}_{\mathrm{rf}}\right.$ ) while holding inlet temperatures, regenerator length, and magnetic material and fluid velocities constant.

A review of Table 5 further emphasizes the importance of maintaining balanced flow (when $\mathrm{mCp}$ of the fluid equals $\mathrm{m}_{\mathrm{C} p}$ of the magnetic material) in the regenerator. A small flow imbalance $(C=0.75)$ results in an $18 \%$ increase in total entropy generation. A larger flow imbalance $(C=0.5)$ can increase total entropy generation by as much as $66 \%$. Earlier magnetic heat pump regenerator design studies did not identify the importance of maintaining a balanced flow in the regenerator. This will impose another design constraint on the concept.

TABLE 5. Integrated Entropy Generation as a Function of the Ratio of mCp

\begin{tabular}{|c|c|c|c|c|c|c|c|c|}
\hline Case & $\begin{array}{l}\text { Regenerator } \\
\text { Length, } \mathrm{m}\end{array}$ & C & $\mathrm{Re}$ & $\begin{array}{l}\dot{S}_{V i} \\
W / K \\
\end{array}$ & $\begin{array}{l}\dot{S}_{\text {Th }} \\
\text { W/K }\end{array}$ & $\begin{array}{l}\text { SThrf }_{\text {W/K }} \\
\end{array}$ & $\begin{array}{l}\dot{S}_{\text {Lostw. }} \\
W / K \\
\end{array}$ & $\begin{array}{l}\dot{S}_{T \circ t}{ }^{\prime} \\
W / K\end{array}$ \\
\hline 1 & 0.28 & 0.25 & 2410 & 0.054 & 1.38 & 2.15 & 0.00 & 3.59 \\
\hline 2 & 0.28 & 0.50 & 1413 & 0.019 & 0.830 & 1.67 & 0.007 & 2.53 \\
\hline 3 & 0.28 & 0.75 & 1081 & 0.013 & 0.537 & 1.20 & 0.045 & 1.80 \\
\hline 4 (Base) & 0.28 & 1.00 & 915 & 0.011 & 0.394 & 0.958 & 0.158 & 1.52 \\
\hline 5 & 0.28 & 2 & 666 & 0.008 & 0.253 & 0.778 & 1.15 & 2.19 \\
\hline 6 & 0.28 & 4 & 541 & 0.008 & 0.122 & 0.463 & 2.46 & 3.05 \\
\hline 7 & 0.28 & 8 & 479 & 0.008 & 0.043 & 0.191 & 3.30 & 3.54 \\
\hline
\end{tabular}


Table 6 presents the results of varying the velocity of the magnetic material and regenerator fluid while holding inlet temperatures, ratio of $\dot{m} \mathrm{C} p$, and regenerator length constant.

The results presented in Table 6 show that entropy generation increases monotonically with increasing Reynolds number. This was expected because increasing Reynolds number while the ratio of $\dot{m} C p$ is held constant results in an increased velocity for the magnetic material. This increases the heat transferred in the regenerator. Therefore, the magnitude of $\dot{S}_{T h m m}$ will be the result of two competing effects; the large increase in heat transfer will increase entropy generation while a small decrease in entropy generation will result from the velocity related increase in the convective heat transfer coefficient. The net result is a monotonic increase in SThmm.

The evaluation of the flow disrupter consisted of two studies. First, a simple flow disrupter was evaluated by determining the integrated local entropy generation as a function of the fraction of flow area blocked by the disrupter. The second evaluation investigated the distribution of local entropy generation to determine if the simple flow disrupter geometry could be improved.

\subsubsection{Analysis of Flow Disrupter Concept}

The results of the evaluation of integrated local entropy generation for a simple flow disrupter design are presented in Table 7 . While the fraction of the flow area blocked by the disrupter was allowed to vary, the mass flow rate of the regenerator fluid (assumed to be water) and the wall heat flux were held constant.

TABLE 6. Integrated Energy Generation as a Function of Regenerator Length

\begin{tabular}{|c|c|c|c|c|c|c|c|c|}
\hline Case & $\begin{array}{l}\text { Regenerator } \\
\text { Length, } \mathrm{m}\end{array}$ & $\underline{c}$ & $\mathrm{Re}$ & $\begin{array}{l}\dot{S}_{V i, s} \\
W / K \\
W^{\prime}\end{array}$ & $\begin{array}{l}\dot{S}_{\text {Than }} \\
W / K\end{array}$ & $\begin{array}{l}\dot{S}_{\text {Thrf, }} \\
W / K\end{array}$ & $\begin{array}{l}\dot{S}_{\text {Lostum }} \\
W / K \\
\end{array}$ & $\begin{array}{l}\dot{S}_{\text {Total }} \\
\text { W/K }\end{array}$ \\
\hline 1 & 0.28 & 1 & 250 & 0.00 & 0.038 & 0.091 & 0.009 & 0.138 \\
\hline 2 & 0.28 & 1 & 500 & 0.00 & 0.138 & 0.332 & 0.047 & 0.517 \\
\hline 3 & 0.28 & 1 & 915 & 0.011 & 0.394 & 0.958 & 0.158 & 1.52 \\
\hline 4 & 0.28 & 1 & 2000 & 0.051 & 1.33 & 3.26 & 0.940 & 5.58 \\
\hline
\end{tabular}


TABLE 7. Integrated Local Entropy Generation for a Simple Flow Disrupter Design as a Function of Blocked Flow Area

\begin{tabular}{|c|c|c|c|c|c|}
\hline Case & $\begin{array}{c}\text { Flow Area Blocked } \\
\text { by Disrupter, \% }\end{array}$ & $\begin{array}{l}\text { SVis } \\
W / K \times 10^{-3} \\
\end{array}$ & $\begin{array}{r}\text { SThrf } \\
W / K \times 10^{-3} \\
\end{array}$ & $\begin{array}{r}S_{T \circ t a} \\
W / K \times 10^{-3} \\
\end{array}$ & $\begin{array}{c}\text { STotal } \\
\text { Near Wall, }\end{array}$ \\
\hline 1 & 0 & 0.004 & 3.939 & 3.943 & 33 \\
\hline 2 & 25 & 0.045 & 3.50 & 3.545 & 37 \\
\hline 3 & 50 & 0.092 & 2.350 & 2.442 & 49 \\
\hline 0 & 75 & 0.461 & 0.892 & 1.353 & 72 \\
\hline
\end{tabular}

Table 7 shows that the disrupter achieves the desired reduction in total entropy generation. When $75 \%$ of the flow area is blocked, the integrated local entropy generation is approximately $1 / 3$ of that encountered without a disrupter (case 1). The improvement was achieved by a $78 \%$ reduction in heat transfer related entropy generation while viscous dissipation related entropy generation increased by $12,000 \%$. It is anticipated that additional improvements in performance could be achieved by further increases in the blocked flow area.

A second interesting item is noted in the last column on Table 7. This reports the percent of the total entropy that is generated in the row of computational cells closest to the wall. As the disrupter size is increased, a progressively larger fraction of the entropy is being generated close to the wal1. This is caused by thinning of the thermal boundary layer resulting in large thermal gradients close to the wall. The concentration of entropy generation close to the wall suggests that finer noding close to the wall may be required to attain a firm understanding of the structure of the local entropy generation.

A review of the distribution of local entropy generation showed that entropy generation caused by heat transfer is concentrated close to the wall in the thermal boundary layer. Total local entropy generation is dominated by heat transfer related entropy generation, resulting in total entropy generation also being concentrated in the thermal boundary layer. Local 
entropy generation caused by viscous dissipation is more evenly distributed throughout the flow field with a significant concentration in the region immediately upstream of the flow disrupter. In this region, the fluid is rapidly decelerating and the strong velocity gradients caused a concentration in local entropy generation. The identification of the upstream concentration in local entropy generation suggested that a more streamlined design should be considered.

While the simulation results discussed above indicate that the flow disrupter may be an attractive heat transfer enhancement technique, such simulation is no substitute for experimental confirmation. It is recommended that an experimental proof-of-concept test be conducted on the flow disrupter concept.

\subsubsection{Figures-of-Merit for Heat Exchanger Analysis}

Researchers involved in evaluating heat transfer enhancements are often faced with the problem of combining pressure drop and heat transfer losses in one figure-of-merit. This has led to the development of several methods for measuring thermodynamic performance of heat transfer enhancements. An example is the efficiency index presented by Webb, Eckert and Goldstein (1971). The definition of the efficiency index is given in Equation (14).

$$
\eta=\frac{S t_{e} / S t_{p}}{f_{e} / f_{p}}
$$

If the efficiency index is greater than 1.0, the enhancement is attractive because the improvement in heat transfer $\left(S t_{e} / S t_{p}\right)$ is larger than the increase in pressure drop $\left(f_{e} / f_{p}\right)$. Unfortunately, a thermodynamic figure-of-merit that does not include second law effects will not, in many cases, give appropriate design guidance.

When we consider internal duct flows, one second law measure of the performance of an enhancement is given by the augmentation entropy generation number $N$ (Bejan 1982) where $N$ is given by Equation (15). An enhancement is attractive when $N<1.0$. 


$$
N=\frac{\left(\frac{S t_{p}}{S t_{e}}\right)\left(\frac{D_{e}}{D_{p}}\right)+\phi\left(\frac{f_{e}}{f_{p}}\right)\left(\frac{D_{p}}{D_{e}}\right)\left(\frac{A_{p}}{A_{e}}\right)^{2}}{1+\phi}
$$

The expression for $N$ given in Equation (15) has two interesting features; the impact of the enhancement concept on heat transfer losses (the term containing $\mathrm{St}$ ) and pressure drop losses (the term containing f) are additive and the relationship between the two terms depends on the irreversibility distribution ratio $\phi$, which is defined by Equation (16).

$$
\phi=\frac{\dot{S}_{v i s}}{\dot{S}_{T h m m}}
$$

$\phi$ gives an indication of the relative importance of the two loss mechanisms. When pressure drop losses are small, $\phi=0 ; N$ is only influenced by heat transfer related losses. The major limitation on the efficiency index [Equation (14)] is that it does not include a measure of the relative importance of heat transfer and pressure drop related losses. The evaluation of entropy generation in the regenerator of a magnetic heat pump is a good example. We will show that Equation (14) predicts that the inclusion of flow disrupters is not attractive while, as we have reported, a second law approach clearly indicates that flow disrupters will result in a significant reduction in thermodynamic losses.

Bejan (1982) shows that for duct flows, Equations (17) and (18) can be written for entropy generation caused by heat transfer $\left(\dot{S}_{\text {Thrf }}\right)$ and viscous dissipation $\left(\dot{S}_{\text {vis }}\right)$.

$$
\dot{S}_{\text {Thrf }}=\frac{L \dot{q}_{o}^{\prime 2}}{4 T^{2} \dot{m} C p} \frac{D_{e}}{S t_{e}}
$$




$$
\dot{S}_{\text {vis }}=\frac{L f_{e}}{D_{e} A_{e}{ }^{2}} \frac{\left(2 \dot{m}^{3}\right)}{\rho^{2} T}
$$

Equations (17) and (18) can be written for the base case and the enhanced case, assuming that $\dot{q}_{0}^{\prime 2}, T, \dot{m}, c p$ and $\rho$ are not influenced by the enhancement, they can be combined to give Equations (19) and (20).

$$
\begin{aligned}
& \frac{S t_{p}}{S t_{e}}=\left(\frac{D_{p}}{D_{e}}\right)\left(\frac{\dot{S}_{\text {Thrf }}}{\dot{S}_{\text {Thrf }}}\right) \\
& \frac{f_{e}}{f_{p}}=\left(\frac{D_{e}}{D_{p}}\right)\left(\frac{A_{e}}{A_{p}}\right)^{2}\left(\frac{\dot{S}_{\text {vis }}}{\dot{S}_{\text {vis }}}\right)
\end{aligned}
$$

It is now possible to use the entropy generation results for the flow disrupter to calculate the ratios of Stanton number and friction factor. We will consider two cases. First, the flow area and hydraulic diameter will be based on the flow path around the disrupter. In the second case, the flow areas and hydraulic diameter will be based on the full width of the flow path without disrupters. In both cases the entropy generation results were taken from Table 4 for a disrupter design that blocks $75 \%$ of the flow area.

Case 1

$$
\begin{aligned}
f_{e} / f_{p} & =1.80 \quad S t_{e} / S t_{p}=1.10 \\
\eta & =1.10 / 1.80=0.611
\end{aligned}
$$

Case 2

$$
\begin{gathered}
f_{e} / f_{p}=115.2 \quad S t_{e} / S t_{p}=4.419 \\
\eta=4.419 / 115.2=0.0383
\end{gathered}
$$


Augmentation Entropy Generation Number

$$
N=1.353 / 2.942=0.343
$$

In both cases, the efficiency index indicates that the use of flow disrupters is not attractive, while $N$ indicates that this enhancement technique is quite attractive. The reason for the different conclusions is related to the distribution of losses. In the base magnetic heat pump regenerator design, thermal losses are two orders of magnitude larger than the pressure drop losses. Consequently, an enhancement technique that reduces heat transfer losses is attractive, even if it results in a substantial increase in pressure drop losses. The efficiency index does not account for the difference in relative importance between the heat transfer losses and pressure drop losses. 


\subsection{EVALUATION OF NUMERICAL ERRORS USING LOCAL ENTROPY GENERATION ANALYSIS}

Numerical modeling of a real thermal process will introduce errors related to the numerical approach. These errors can be considered as sources of numerical entropy generation. Typical sources of numerical entropy generation include numerical viscosity, round-off errors, truncation errors, and numerical instabilities. As Arglow, Emanual, and Rasmussen (1987) observed, for a valid simulation, numerical entropy generation (entropy generation caused by the numerical approach) should be small compared to the natural entropy generation (entropy generation caused by the physics of the phenomena). While a number of investigators have developed relationships for natural entropy generation (Bejan 1979a; Argrow, Emanual, and Rasmussen 1987), a literature review did not identify procedures for calculation of numerical entropy generation. The current evaluation of local entropy generation suggests that numerical entropy generation can be characterized using a comparison of local entropy generation with the results of an entropy balance.

\subsection{THE ENTROPY BALANCE}

The procedure described in Section 3 calculates local entropy generation based on the local gradients and fluid properties. Entropy generation can also be calculated by conducting an entropy balance on a region. Applying the second law of thermodynamics to an open thermodynamic system without shaft work results in the entropy balance equation [Equation (21)].

$$
\iiint \dot{s}_{\text {gen }}=\iint \frac{\dot{q}^{\prime \prime}}{T}+\sum_{\text {out }} \mathrm{ms}-\sum_{\text {in }} \mathrm{ms}
$$

As Equation (21) shows, the volume integral of the local entropy generation should equal the summation of entropy flows into and out of the open system.

The entropy balance equation can be evaluated using the results from typical CFD calculations and local entropy generation analysis. Local entropy generation integrated over the region of interest gives the term on the lefthand side of Equation (21). A typical CFD code will provide information on 
heat fluxes and temperatures required for the calculation of the surface integral included in the first term on the right-hand side of Equation (21). The remaining two terms are related to the inflow and outflow of entropy associated with mass flow. Typical CFD simulation results allow the calculation of the mass flow rates but the specific entropy may not be available.

The change in specific entropy between two states can be calculated for a simple compressible substance using Equation (22) (Van Wylen and Sonntag 1971)

$$
T d s=d h-v d P
$$

The evaluation of Equation (22) requires an equation of state and a functional relationship between the constant pressure specific heat and temperature. By assuming that the flow is incompressible and that specific heats are not temperature dependent, Equation (22) can be integrated between the inlet and outlet states. The relationship is then substituted into Equation (21), resulting in Equation (23).

$$
\iiint \dot{S}_{\text {gen }}=\iint \frac{q^{\prime \prime}}{T}+\sum_{\text {outlet }} m c_{p} \ln \left(\frac{T_{\text {out }}}{T_{\text {ref }}}\right)-\sum_{\text {inlet }} m c_{p} \ln \left(\frac{T_{\text {in }}}{T_{\text {ref }}}\right)
$$

where Tout and Tin are the mean temperatures across the inlet and outlet ports and $T_{r e f}$ is the reference temperature.

For an incompressible fluid with specific heats independent of temperature, entropy generation caused by viscous dissipation results in an increase in the fluid temperature. The TEMPEST computer code does not include the viscous dissipation terms in the energy equation; therefore, it was not possible to compare the local entropy generation rate when it included entropy generation caused by both viscous dissipation and thermal gradients with an entropy balance that did not include the temperature rise caused by viscous dissipation. A meaningful comparison can only be made if the local entropy 
generation and entropy balances are based on entropy generation caused by thermal gradients.

\subsection{RESULTS OF SAMPLE SIMULATIONS}

Three convective heat transfer simulations were used to investigate the relationship between local entropy generation and the entropy balance equation: 1) two-dimensional Poiseuille flow between parallel plates with a constant surface heat flux, 2) two-dimensional laminar entrance region flow with constant surface heat flux, and 3) two-dimensional recirculating flow with heat transfer.

The third simulation was previously selected as a test problem for evaluating numerical models of advection (Smith and Hutton 1982) and will subsequently be referred to an the IAHR (International Association for Hydraulic Research) test problem.

These simulations include a range of features encountered in practical advection-diffusion problems that were suspected to impact the comparison of local entropy generation with an entropy balance. The first case is typical of problems without streamline curvature and moderate thermal gradients. The second simulation includes weak streamline curvature and moderate thermal gradients, while the IAHR test problem has strong streamline curvature and steep thermal gradients.

The first two simulations involve a similar geometry and will be discussed together. The first simulation models a fully developed velocity field between two flat plates but includes a developing temperature profile. The plates are separated by a distance of $0.5 \mathrm{~m}$. The fluid was assumed to be helium entering with a constant temperature of $150^{\circ} \mathrm{C}$. Fluid properties were assumed to be constant and were evaluated at $150^{\circ} \mathrm{C}$. The mass flow rate was $0.0377 \mathrm{~kg} / \mathrm{s}$, while the surface heat flux was $86 \mathrm{~W} / \mathrm{m}^{2}$. The computational region extended over the 34-m length of the flat plates, along the direction of flow. The second simulation involved developing both velocity and temperature profiles. Helium was assumed to enter with a constant velocity in the radial direction and the velocity profile was assumed to evolve as the helium flowed along the 
pipe. The two plates were separated by a distance of $0.5 \mathrm{~m}$ and the helium was assumed to enter at a temperature of $150^{\circ} \mathrm{C}$. The average mass flow rate and surface heat flux was $0.0173 \mathrm{~kg} / \mathrm{s}$ and $8600 \mathrm{~W} / \mathrm{m}^{2}$, respectively. The computational region extended over the first $0.034 \mathrm{~m}$ of length of the flat plates in the direction of flow.

The results of the TEMPEST simulations for the first and second cases are summarized on Table 8. The first column presents the integrated local entropy generation caused by temperature gradients. The second column is the entropy generation calculated using Equation (23), while the third column is the absolute value of the difference between the first and second columns. Theoretically, in the absence of numerical error, the first and second columns should be comparable. It was anticipated that the integrated local entropy generation should be lower than the entropy generation predicted by the entropy balance equation because the entropy balance equation included numerical entropy generation. In addition, it was expected that the difference between integrated local entropy generation and the results of the entropy balance equation should be larger for the second problem, which was expected to include more numerical entropy generation. As Table 8 demonstrates, both trends were confirmed by the sample simulations. Consequently, we interpret the difference between the integrated local entropy generation and the results of the entropy balance equation as being a measure of numerical entropy generation.

The IAHR problem was a more challenging simulation because it involved stronger streamline effects and steep thermal gradients. The IAHR test problem consisted of the solution of a general two-dimensional advection-diffusion

TABLE 8. Comparison of Integrated Local Entropy Generation with the Results of the Entropy Balance Equation for Poiseuille and Entrance Flow

\begin{tabular}{|c|c|c|c|}
\hline No. & $\iiint \mathrm{s}_{\text {gen, }} w / K$ & $S_{\text {gen, }} W / K$ & $\%$ Error \\
\hline 1 & 0.08484 & 0.085 & 0.19 \\
\hline 2 & 0.05842 & 0.0609 & 4.24 \\
\hline
\end{tabular}


equation given by Equation (24) in the rectangular region where the Peclet number was defined based on the grid dimension.

$$
\stackrel{+}{\mathrm{u}} \cdot \nabla \theta-\frac{1}{\mathrm{Pe}} \nabla^{2} \theta=0
$$

The velocity field for the IAHR problem was specified analytically by Equation (25).

$$
u=2 y\left(1-x^{2}\right), \quad v=-2 x\left(1-y^{2}\right)
$$

The boundary conditions for the advected variables were specified over the inlet and perimeters except for the outlet, which was computed. The outlet boundary did include a zero gradient boundary condition. The advected variable profile at the inlet contained a sharp gradient in value approximately midway along the inlet length. The boundary conditions were expressed analytically by Equation (26).

$$
\begin{array}{r}
\theta=1+\tanh [(2 x+1) a] \quad y=0-1 \leq x \leq 0 \text { (inlet) } \\
x=-1 \quad 0 \leq y \leq 1 \\
\theta=1-\tanh a y=1 \quad-1 \leq x \leq 1 \\
x=1 \quad 0 \leq y \leq 1
\end{array}
$$

The constant a included in Equation (26) was assumed to have a value of 10 .

The IAHR problem was simulated by solving the energy equation in TEMPEST with a fixed velocity field and having temperature serve as the advected variable. The energy equation in TEMPEST reduces to Equation (24), when source terms are deleted and constant fluid properties are assumed. The problem was emulated using a uniform $20 \times 10$ grid, shown in-Figure 12 . Figure 12 also includes a depiction of the velocity field by streamlines. The simulation was conducted for a range of Peclet numbers $(10,100,500,1000)$. The Peclet number was varied by modifying the thermal conductivity of the fluid, while holding the other fluid properties constant. 


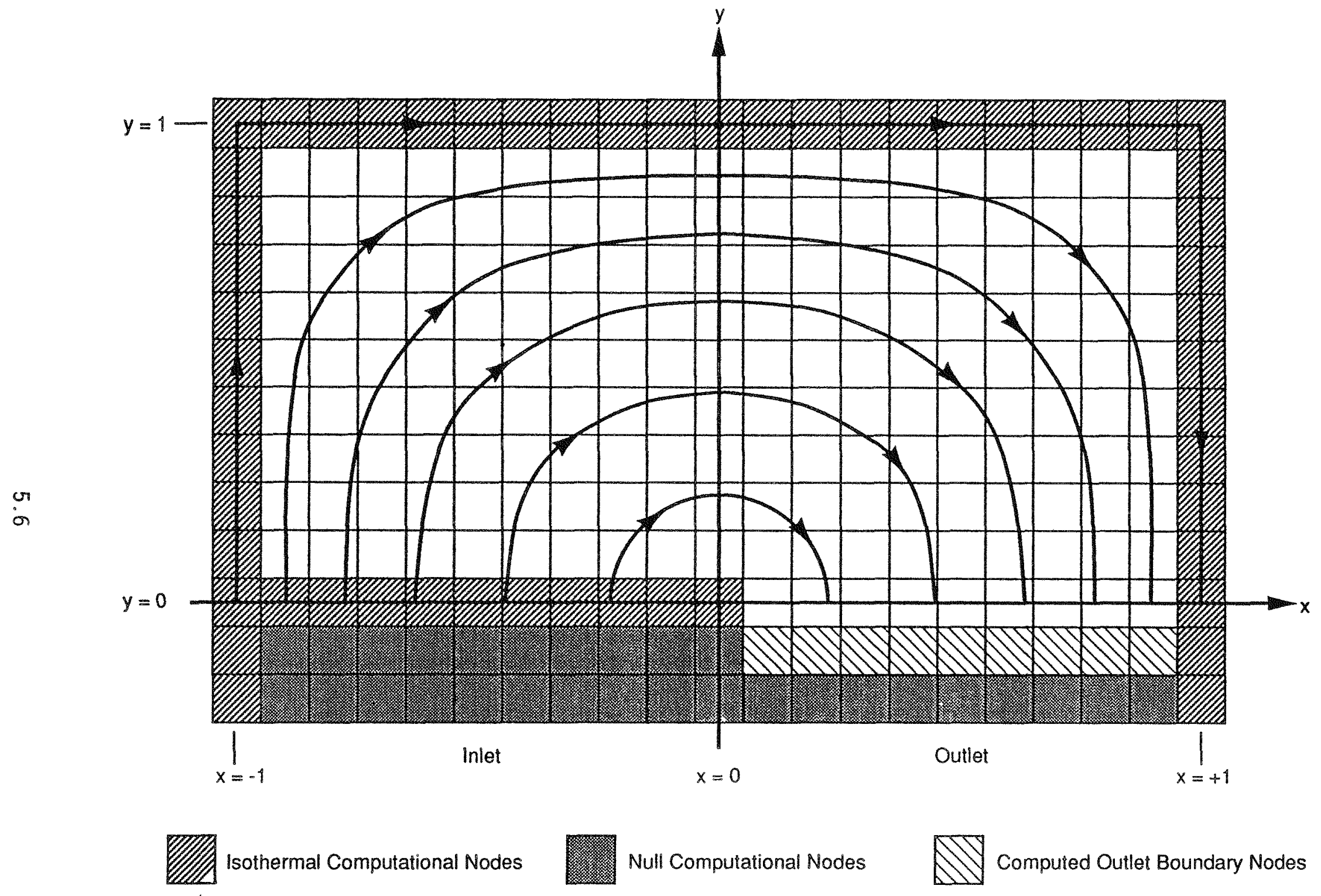

FIGURE 12. Computational Grid for IAHR Test Problem 
Smith and Hutton (1982) considered Schonauer's solution schemes to be sufficiently accurate to serve as a reference solution for evaluating the accuracy of other numerical schemes. Figure 13 presents the outlet profile of the advection variable for both the Schonauer solution (Smith and Hutton 1982) and TEMPEST for each of the Peclet numbers. The general trend is a progressively declining agreement between the TEMPEST solution and the reference solution with increasing Peclet number. At low Peclet numbers (high thermal conductivities), the effects of numerical diffusion are negligible because the thermal conductivity dominated advection. As the Peclet number increased, the contribution of numerical advection to the solution of the temperature field increased resulting in an increasingly inaccurate solution. There was no apparent difference between the TEMPEST results for a Peclet number of 500 and 1000 because the numerical source of diffusion dominated thermal conductivity.

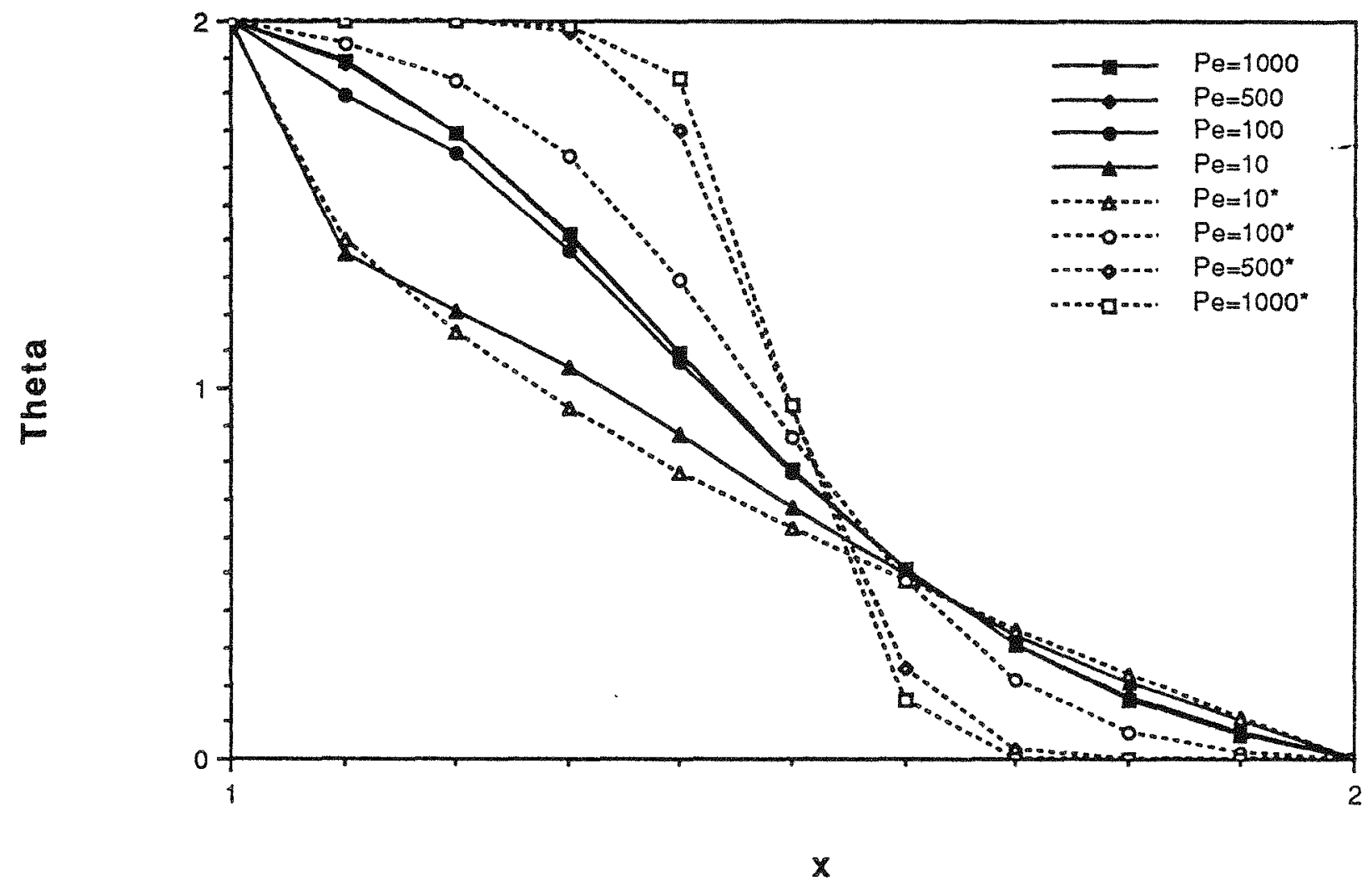

FIGURE 13. Outlet Profile for Advecting Variable 
The results for the IAHR problem are summarized in Table 9. As with the first two simulations, the integrated local entropy generation rates are compared to the results of the entropy balance equation. In all cases, the comparison is poor with the magnitude of the difference between the results approximating the order of magnitude of the entropy balance. It is our hypothesis that the poor comparison is caused by the impact of numerical entropy generation on the results of the entropy balance equation. This assumes that the integrated local entropy generation is correct, but the evaluation of Equation (1) depends on accurate data for the temperature field. When strong numerical diffusion is encountered, the solution for the temperature field will be affected. As Figure 11 shows, at a Peclet number of 10, the exiting temperature profiles for the TEMPEST simulation and the reference results are quite similar, suggesting that the results of the local entropy generation analysis are valid. At higher Peclet numbers, the results of the local entropy generation analysis would have to be used with care.

The comparison of integrated local entropy generation with a surface integration of entropy flows (the entropy balance equation) resulted in two observations. First, the difference between the integration of local entropy generation and the surface integration of entropy flows is a measure of numerical entropy generation. Second, the accuracy of local entropy generation analys is depends on the accuracy of the underlying CFD code. To the extent that the CFD code is producing accurate results, the local entropy generation will be accurately predicted.

TABLE 9. Comparison of Integrated Local Entropy Generation with the Results of the Entropy Balance Equation for IAHR Test Problem

$\begin{array}{rcccc}\text { Pe } & \iiint \dot{S}_{\text {gen, } W / K} & & \dot{S}_{\text {gen, } W / K} & \\ 10 & 0.1060 & & 0.3003 & 0.1943 \\ 100 & 0.01261 & 0.2283 & 0.2157 \\ 500 & 0.002573 & 0.2201 & 0.2175 \\ 1000 & 0.001289 & 0.2173 & 0.2160\end{array}$




\subsection{CONCLUSIONS}

The results of this study confirm the feasibility and usefulness of numerical calculation of local entropy generation in complicated thermal processes. Specific conclusions concerning the feasibility of numerical calculation of local entropy generation include:

- The integration of local entropy generation calculations into an existing CFD code proved to be straightforward and did not significantly increase run times.

- The comparison of numerical and analytical results for a simple benchmarking problem was successful.

- Numerical calculation of local entropy generation and its comparison with a surface integration of entropy flows provides a measure of numerical entropy generation that can be compared to natural entropy generation to judge the validity of the numerical calculations.

Specific conclusions concerning the usefulness of numerical calculation of local entropy generation include:

- Numerical calculations allow the investigation of the structure of local entropy generation in thermal processes. The impinging jet problem is an example. The results show that for the sample problem, entropy generation is concentrated in the boundary layer. To a great extent, understanding the boundary layer will allow an understanding of local entropy generation. This will not necessarily be true for different geometries and more complicated processes.

- Numerical calculations can help a designer optimize a component. The results of the impinging jet problem identified a jet Reynolds number that minimizes entropy generation. This information would be useful to a designer trying to minimize the thermodynamic losses in a jet impingement heat exchanger.

A number of important design guidelines were identified as part of the magnetic heat pump regenerator evaluation. These include:

- In the base case regenerator design, heat transfer related entropy generation dominates viscous dissipation losses. This suggests that the design should be altered to improve heat transfer, even if the alteration results in a substantial increase in viscous dissipation losses.

- It is important to maintain balanced flow (where micp of the magnetic material is approximately equal to $\dot{m}(p)$ of the regenerator fluid) in the regenerator. Substantial deviation from balanced flow conditions can result in a significant increase in thermodynamic losses. 
- Entropy generation caused by conduction in the magnetic material is a significant loss mechanism and must be included explicitly in any performance evaluation.

- The addition of a flow disrupter can significantly improve the performance of the regenerator. The optimum disrupter design will block more than $75 \%$ of the flow area.

The evaluation of the flow disrupter concept illustrates the importance of using second law analysis for evaluating heat transfer enhancements. Typical conventional figures-of-merit such as the efficiency index suggest that the flow disrupter is not an attractive enhancement technique while local entropy generator analysis shows that the use of flow disrupters is very attractive. The reason for the different conclusions is related to the distribution of losses. In the base case magnetic heat pump regenerator design, thermal losses are two orders of magnitude larger than the pressure drop losses. Consequently, an enhancement technique that reduces heat transfer losses is attractive, even if it results in a substantial increase in pressure drop losses. The efficiency index is a first law figure-of-merit and does not account for the difference in relative importance between the heat transfer losses and the pressure drop losses.

Based on the results of this study, we have made several recommendations for future research.

- Given the simplicity and usefulness of including local entropy generation calculations in CFD codes, CFD code developers should be encouraged to incorporate local entropy generation calculation in a wide range of simulation tools.

- Local entropy generation calculations should be expanded to involve more complicated processes including multiple phases, multiple components, reacting flows, compressible flows, and radiation heat transfer. 


\subsection{REFERENCES}

Argrow, B. M., G. Emanual, and M. L. Rasmussen. 1987. "Entropy Production in Nonsteady General Coordinates." AIAA Journal 25(12):1629-1631.

Barclay, J. A., 1984, "A Comparison of Gas and Magnetic Refrigeration." In Proceedings of 22nd Heat Transfer Conference (Niagara Falls, New York, August 1984). Los Alamos National Laboratory, Los Alamos, New Mexico.

Barclay, J. A., 1987, "Magnetic Refrigeration: A Review of a Developing Technology." (Presented at the Cryogenics Engineering Conference in St. Charles, Illinois, June 1987.) Astronautics Corporation of America, Madison, Wisconsin.

Barclay, J. A., and S. Sarangi, 1984, "Selection of Regenerator Geometry for Magnetic Refrigerator Applications." In Cryogenic Processes and Equipment Proceedings of ASME Cryogenic Symposium December 1984. American Society of Mechanical Engineers, New York, New York.

Bejan, A. 1979a. "A Study of Entropy Generation in Fundamental Convective Heat Transfer." J. Heat Transfer 101:718-725.

Bejan, A. 1979b. "Second Law Analysis in Heat Transfer." (Presented at workshop on "The Second Law of Thermodynamics", George Washington University, Washington, D.C., August 14-16, 1979.) Duke University, Durham, North Carolina.

Bejan, A. 1982. Entropy Generation Through Heat and Fluid Flow. Wiley Interscience, New York, New York.

Drost, M. K., and J. R. Zaworski. 1988. "A Review of Second Law Analysis Techniques Applicable to Basic Thermal Science Research." In Thermodynamic Analys is of Chemically Reactive Systems, pp. 7-12. American Society of Mechanical Engineers, New York, New York.

Dunbar, W. R. 1982. Computer Simulation of a High Temperature Solid Electrolyte Fuel Cel1. M.S. Thesis, Marquette University, Milwaukee, Wisconsin.

El-Sayed, Y. 1988. "On Exergy and Surface Requirements for Heat Transfer Processes Involving Binary Mixtures." In Second Law Analys is in Heat/Mass Transfer and Energy Conversion, Pp. 19-24. American Society of Mechanical Engineers, New York, New York.

Gaggioli, R. A. 1983. "Second Law Analys is for Process and Energy Engineering." In Efficiency and Costing, Second Law Analysis of Processes. ACS 235, American Chemical Society, Washington, D.C.

Hutchinson, R. A., and S. E. Lyke. 1987. "Microcomputer Analys is of Regenerative Heat Exchangers for Oscillating Flows." In Proceedings: 1987 
ASME/JSME Thermal Engineering Joint Conference, Vo1. 2, pp. 653. American Society of Mechanical Engineers, New York, New York.

Kays, M. W., and M. E. Crawford. 1980. Convective Heat and Mass Transfer. 2nd ed., McGraw-Hill Company, New York, New York.

Kirol, L. D., J. I. Mills, K. S. Fullmer and J.N. Zabriskie. 1984. Magnetic Heat Pump Feasibility Assessment. EGG-2343, Idaho National Engineering Laboratory, Idaho Falls, Idaho.

Liang, H., and T. H. Kuehn. 1988. "Irreversibility Analys is of a Water to Water Mechanical Compression Heat Pump." In Analysis and Applications of Heat Pumps, pp. 31-36. American Society of Mechanical Engineers, New York, New York.

Smith, R. M., and A. G. Hutton. 1982. "The Numerical Treatment of Advection: A Performance Comparison of Current Methods." Numerical Heat Transfer 5:439461 .

Trent, D. S., L. L. Eyler, and M. J. Budden. 1989a. TEMPEST - A Three-Dimensional Time-Dependent Computer Program for Hydrothermal Analys is, Volume 1: Numerical Methods and User Input. PNL-4348, Vol. 1, Rev. 2, Pacific Northwest Laboratory, Richland, Washington.

Trent, D. S., L. L. Eyler, and M. J. Budden. 1989b. TEMPEST - A ThreeDimensional Time-Dependent Computer Program for Hydrothermal Analysis, Volume 2: Assessment and Verification Results. PNL-4348, Vol. 2, Rev. 2, Pacific Northwest Laboratory, Richland, Washington.

Van Wylen, J.G., and R. E. Sonntag. 1973. Fundamentals of Classical Thermodynamics. John Wiley and Sons, Inc., New York, New York.

Webb, R. L., E. R. G. Eckert, and R. L. Goldstein. 1971. "Heat Transfer and Friction in Tubes with Repeated-Rib Roughness." Int. J. Heat Mass Transfer $14: 601-617$.

White, M. D., and M. K. Drost. 1989. "Numerical Prediction of Local Entropy Generation in an Impinging Jet." In Analys is and Design of Energy Systems: Fundamentals and Mathematical Techniques, AES-Vol 5. American Society of Mechanical Engineers, New York, New York. 


\section{DISTRIBUTION}

No. of

Copies

OFFSITE

U.S. Department of Energy

Attn: M. Gunn

Energy Conversion and Utilization Technology Program

Forrestal B1dg., CE-12 5E-066

Washington, DC 20585

12 DOE/Office of Scientific and

Technical Information

Argonne National Laboratory

Attn: T. Thomas

9700 South Cass Avenue

Argonne, IL 60439

Astronautics Corp. of America

Attn: J. Barclay

Astronautics Technology Center

5800 Cottage Grove Rd.

Madison, WI 53716

Duke University

Attn: A. Bejan

Department of Mechanical Eng. and Material Science

Durham, NC 27706

R. A. Gaggioli

391 High Street

Westwood, MA 02090

Idaho National Engineering Lab.

Attn: J. Mills

PO Box 1625

Idaho Falls, ID 83415

Mississippi State University

Attn: G. A. Adebiyi

Mechanical and Nuclear Eng. Dept.

Mississippi State, MS 39762
No. of

Copies

Oregon State University

Attn: G. M. Reistad

Mechanical Engineering Dept.

Corvallis, OR 97331

Oregon State University

Attn: J. Zaworski

Mechanical Engineering Dept.

Corvallis, OR 97331

The George W. Woodruff School of Mechanical Engineering

Attn: W. J. Wepfer

Georgia Institute of Technology

225 North Ave. NW

Atlanta, GA 30332

ONSITE

DOE Richland Operations Office

R. B. Goranson, $A 5-90$

19 Pacific Northwest Laboratory

M. K. Drost, K5-21 (10)

B. M. Johnson, K5-25

L. D. Kannberg, K5-21

G. L. Work, K2-51

Technical Report Files (5)

Publishing Coordination

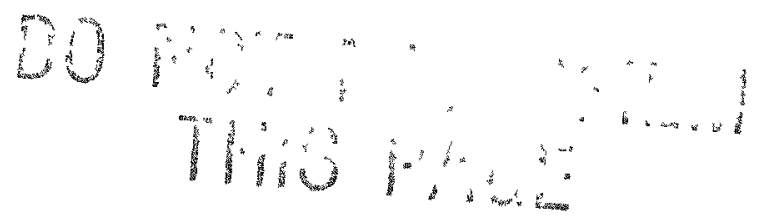

Distr.1 